\title{
The role of fundamental solution in Potential and Regularity Theory for subelliptic PDE
}

\author{
Andrea Bonfiglioli, Giovanna Citti, Giovanni Cupini, Maria Manfredini, \\ Annamaria Montanari, Daniele Morbidelli, Andrea Pascucci, Sergio Polidoro, \\ Francesco Uguzzoni
}

\author{
tu se' lo mio maestro e 'l mio autore \\ tu se' solo colui da cu' io tolsi \\ lo bello stilo che m'ha fatto onore. \\ Dante Alighieri
}

\begin{abstract}
In this survey we consider a general Hörmander type operator, represented as a sum of squares of vector fields plus a drift and we outline the central role of the fundamental solution in developing Potential and Regularity Theory for solutions of related PDEs. After recalling the Gaussian behavior at infinity of the kernel, we show some mean value formulas on the level sets of the fundamental solution, which are the starting point to obtain a comprehensive parallel of the classical Potential Theory. Then we show that a precise knowledge of the fundamental solution leads to global regularity results, namely estimates at the boundary or on the whole space. Finally in the regularity problem of non linear differential equations we use an ad hoc modification of the parametrix method, based on the properties of the fundamental solution of an approximating problem.
\end{abstract}

\footnotetext{
Andrea Bonfiglioli, Giovanna Citti, Giovanni Cupini, Maria Manfredini, Annamaria Montanari, Daniele Morbidelli, Andrea Pascucci, Sergio Polidoro, Francesco Uguzzoni

Dipartimento di Matematica, Università di Bologna, e-mail: andrea.bonfiglioli6e unibo.it, giovanna.citti@unibo.it, giovanni.cupini@unibo.it, maria.manfredini@unibo.it, annamaria.montanari@unibo.it, daniele. morbidelli@unibo.it, andrea.pascucci@unibo.it, francesco.uguzzoni@ unibo.it

Sergio Polidoro

Dipartimento di Scienze Fisiche, Informatiche e Matematiche e-mail: sergio.polidoro@ unimore.it
} 


\section{Introduction}

In this paper we consider a general operator of the form

$$
L_{A}=\sum_{i, j=1}^{m} a_{i, j}(t, x) X_{i} X_{j}-X_{0}
$$

where

$$
X_{i}=\sum_{j=1}^{N} \sigma_{i, j} \partial_{x_{j}}, \quad X_{0}=\partial_{t}+\sum_{j=1}^{N} \sigma_{0, j} \partial_{x_{j}},
$$

and the coefficients $\sigma_{i, j}$ will be dependent only on the spatial variables $x \in \mathbb{R}^{N}$. We also require that $X_{0}, X_{1}, X_{2}, \ldots, X_{m}$ is a system of real smooth vector fields defined in some domain $D \subset\left[0, T\left[\times \mathbb{R}^{N}\right.\right.$ satisfying the Hörmander's rank condition at any point:

$$
\operatorname{rank}\left(\operatorname{Lie}\left(X_{0}, \ldots, X_{m}\right)(t, x)\right)=N+1, \quad \forall(t, x) \in D .
$$

The matrix $A=\left\{a_{i, j}(t, x)\right\}_{i, j=1}^{m}$ is real symmetric and uniformly positive definite, that is

$$
\lambda^{-1}|\xi|^{2} \leq \sum_{i, j=1}^{m} a_{i, j}(t, x) \xi_{i} \xi_{j} \leq \lambda|\xi|^{2}
$$

for some $\lambda>0$ and for every $\xi \in \mathbb{R}^{N}$ and every $(t, x) \in D$. We will assign degree 1 to the vector fields $\left(X_{i}\right)_{i=1, \ldots, m}$, (denoted $d\left(X_{i}\right)=1$ ), while $d\left(X_{0}\right)=2$. We will denote $d((t, x),(\tau, \xi))$ the Carnot-Carathéodory metric generated in $D$ by the vector fields $X_{0}, X_{1}, \ldots, X_{m}$ with their degrees. Precisely for every pair of points $(t, x)$ and $(\tau, \xi)$, we define

$$
\begin{aligned}
& d((t, x),(\tau, \xi))=\inf \{r>0 \mid \text { there is a Lipschitz path } \gamma \text { such that } \\
& \gamma(0)=(t, x), \quad \gamma(1)=(\tau, \xi), \text { and, for a.e. } s, \gamma(s)=\sum_{i=0}^{m} \beta_{i}(s) X_{i}(\gamma(s)) \\
& \text { with } \left.\left|\beta_{i}(s)\right| \leq r \text { for } i=1, \ldots, m, \text { and }\left|\beta_{0}(s)\right| \leq r^{2}\right\}
\end{aligned}
$$

The Carnot-Carathéodory metric generated by the vector fields $X_{0}, X_{1}, \ldots, X_{m}$ plays a crucial role in the regularity theory for subelliptic degenerate operators.

After the celebrated Hörmander's paper [65], where the explicit fundamental solution of a Kolmogorov-type operator was calculated, Folland [55], RothschildStein [98], Jerison and Sanchez Calle [66] proved existence and asymptotic behavior of the fundamental solution, under the assumption that $A$ is the identity. Almost at the same time, Franchi and Lanconelli [58] studied regularity of sum of squares of diagonal vector fields and established a Poincaré type inequality. The equivalence of several distances was proved by Nagel, Stein and Wainger in [88]. After that, in the last twenty years we witnessed an extraordinary development of the theory of subelliptic operators. We refer the reader to the book [21] and to the introduction of each section of this paper, for more historical remarks and references. 
A significant contribution to the development of Potential Theory of subelliptic PDEs is due to Ermanno Lanconelli. His personal and original approach is based on a far-reaching use of the fundamental solution in order to prove, in this setting, a complete parallel of the classical Potential and Regularity Theory.

In this paper we take this perspective, and we describe from a unitary point of view a number of results obtained by the authors in collaboration with him. In Section 2 we will recall Gaussian estimates of the fundamental solution of large classes of operators of the type (1). In particular for the heat equation we discuss the results of Bonfiglioli, Lanconelli and Uguzzoni [19], [20], Bramanti, Brandolini, Lanconelli and Uguzzoni in [28], and for the Kolmogorov operator we quote the results of Polidoro [95], Lanconelli and Polidoro [78], Lanconelli and Pascucci in [74]. In Section 3 we describe the quasi-exponential mappings, introduced in Lanconelli and Morbidelli [73], which are a tool to obtain a Poincaré inequality. Level sets of the fundamental solution are special families of balls, on which mean value formulas have been proved by Citti, Garofalo and Lanconelli (see [41]), Lanconelli and Pascucci (see [75]), which lead to another proof of the Poincaré inequality. Using the mean value formulas, characterizations of subharmonicity were obtained by Bonfiglioli and Lanconelli ([17], [13], [15], [17]). The optimality of these sets have been investigated by Lanconelli [72], Abbondanza and Bonfiglioli [1], Kogoj, Lanconelli and Tralli [68], Kogoj and Tralli [69]. The properties of the fundamental solution immediately imply internal regularity of solutions. Here we are also interested in global regularity of solutions which will be presented in Section 4. Precisely we will recall Schauder regularity up to the boundary, by Manfredini in [79], and the estimates on the whole of space by Bramanti, Cupini, Lanconelli and Priola [31] and [32]. Finally in Section 5 we conclude our survey with a discussion on regularity of solutions of non linear-equations with nonlinearity in the vector fields, and in particular of the Levi equation. For the case of $\mathbb{C}^{2}$ see [40], [42]. For the Levi equation in $\mathbb{C}^{n+1}$ with $n>1$, we refer to the regularity results in [83], [81], [49], the counterexamples by Gutierrez, Lanconelli and Montanari [62], and the symmetry results by Martino and Montanari [80].

\subsection{Applications to complex analysis, finance and vision}

Equation (1) is a natural generalization of the classical equation which models particle interactions in phase spaces. In this case the drift term expresses the coupling position-velocity:

$$
X_{0}=\sum_{j=1}^{m} p_{j} \partial_{q_{j}}+\partial_{t}
$$

and the matrix $\left(a_{i j}\right)$ is the identity in the space of velocities:

$$
L=\frac{1}{2} \sum_{j=1}^{m} \partial_{p_{j}}^{2}-\sum_{j=1}^{m} p_{j} \partial_{q_{j}}-\partial_{t}, \quad(t, q, p) \in \mathbb{R} \times \mathbb{R}^{m} \times \mathbb{R}^{m}
$$


Kolmogorov constructed already in 1934 an explicit fundamental solution of (4) (see (13) below) which is a $C^{\infty}$ function outside the diagonal [70].

As it is well known in this problem the propagation is expressed as a $2 \mathrm{~m}$ dimensional stochastic process $Y=(P, Q)$, and the fundamental solution of (4) describes its transition density. As a result of the random collision, the propagation of the $P$-variables is driven by a $m$-dimensional standard Brownian motion $W$, while $Q$ variables are related to the $P$ by a natural differential equality. Then the propagation is formalized as solution of the Langevin's equation

$$
\left\{\begin{array}{l}
d P(t)=d W(t), \\
d Q(t)=-P(t) d t .
\end{array}\right.
$$

In the deterministic expression, the differential relation between the variables is coded as a 1-form. Clearly the fundamental solution of the more general equation (1) has an analogous probabilistic meaning.

These models, introduced at microscopical level for the description of kinetic theory of gases (see [36]), can be applied at meso-scopical level in biological models, where the atoms are replaced by cells. Indeed simple cells of the cortex are able to detect not only the intensity of the visual input, but also secondary variables, typically gradient of perceived images or velocities of objects. The differential relationa between these variables allow to identify the cortical space as a phase space, and to describe propagation of the visual signal with instruments similar to the ones recalled above. Consequently propagation of the signal have been modeled with a Kolmogorov-Fokker-Planck equation by [87], [109], [3], models with non linear differential equations are due to [45].

Also in financial mathematics, stochastic models involving linear and non linear Kolmogorov type equations are relevant because they appear when considering path-dependent contingent claims (see, for instance, [91]). More precisely, let us assume that the price $S$ of an asset is defined as in the Black-Scholes framework [10]: $S_{t}=\exp \left(\left(r-\frac{\sigma^{2}}{2}\right) t+\sigma W_{t}\right)$ where $r$ and $\sigma$ denote the constant interest rate and volatility respectively. Then the price $u=u\left(t, S_{t}, Y_{t}\right)$ of a contingent claim which depends on $Y_{t}=\int_{0}^{t} \log S_{s} d s$, solves a Kolmogorov type equation (see, for instance, [4]). Other examples of path-dependent models arising in finance can be found in [63] and [56].

As a generalization of the phase space, we can consider a general CR structure or a real hyper-surface in $\mathbb{C}^{n}$ : in this case the analogous of the coupling positionvelocity is realized by the quasicomplex structure. The basis of the complex tangent bundle is a lower dimensional distribution, described by a family of vector fields. In particular, curvature equations are naturally expressed in terms of vector fields and provide examples non linear Hörmander type PDE (see [39, 84]). 


\section{Fundamental solutions of linear operators}

The first aspect of the problem we want to face is the existence and Gaussian estimates and of a fundamental solution for the operator of (1) with Hölder continuous coefficients. The first existence results for operators of Hörmander type operators, refer to sum of squares of vector fields, plus a drift term.

$$
L_{I}=\sum_{i=1}^{m} X_{i}^{2}-X_{0}
$$

In this case the matrix $\left(a_{i j}\right)$ in $\sqrt{1}$ is the identity. In particular Hörmander pointed out in the introduction of his celebrated paper on hypoelliptic second order differential equations [65] that the Kolmogorov method can also be applied to a class of operators which generalize equation (4), but fall in the general framework (1). Uniform but not Gaussian estimates, for families of Hörmander operators of this type, were proved by Rothschild and Stein [98]. Gaussian but not uniform estimates were proved by Jerison and Sánchez-Calle [66], via Gevrey regularity methods, Varopoulos, Saloff-Coste and Coulhon [107], via semi-group theory, and by Kusuoka and Stroock [71], via probabilistic techniques.

The results we plan to present here refer to non divergence form operators, with $C^{\alpha}$ coefficients, and the main results regarding the heat equation are due to Bonfiglioli, Lanconelli and Uguzzoni [19], [20], Bramanti, Brandolini, Lanconelli and Uguzzoni in [28] while for the Kolmogorov operator we quote the results of Polidoro [95], Lanconelli and Polidoro [78], Lanconelli and Pascucci in [74].

The contribution of these papers are twofold: from one side they establish uniform Gaussian bounds for the fundamental solution of a model operator of the form

$$
L_{w}=\sum_{i, j=1}^{m} a_{i j}(w) X_{i, w} X_{j, w}-X_{0, w}
$$

where $\left(a_{i j}(w)\right)$ are constant coefficients while the family $\left(X_{i, w}\right)$ is can be the given operators or a nilpotent and stratified approximation. This goal can be reached either with probabilistic instruments or with an analytic approach:

They apply the Levi's parametrix method to prove the results for operators with Hölder continuous coefficients $a_{i j}$. The method is based on the approximation of the fundamental solution $\Gamma_{A}(z ; \zeta)$ of the given operator by the fundamental solution $\Gamma_{w}(z ; \zeta)$ of a model operator belonging of the previous studied class and obtained by evaluating the coefficient at a point $w$ and approximating the vector fields in a neighborhood of each point $w$.

We present here the application of the method in two particularly significant cases of equation (1): the Kolmogorov equation, which will be studied with stochastic instruments and the heat equation, which will be studied with deterministic ones. 


\subsection{Kolmogorov type operators}

We will call Kolmogorov type operators an operator of the form

$$
L_{A} u(t, x):=\sum_{i, j=1}^{m} a_{i j}(t, x) \partial_{x_{i} x_{j}} u(t, x)+\sum_{i, j=1}^{N} b_{i j} x_{j} \partial_{x_{i}} u(t, x)-\partial_{t} u(t, x)
$$

where $a_{i j}$ satisfy condition (2). This operator clearly falls in the general framework of equation 11 , by choosing $X_{0}=\partial_{t}-\sum_{i, j=1}^{N} b_{i, j} x_{i} \partial_{x_{j}}, X_{j}=\partial_{j}$. In order to study its fundamental solution, we will preliminary study a model operator

$$
K u(t, x):=\frac{1}{2} \sum_{j=1}^{m} \partial_{x_{j}}^{2} u(t, x)+\sum_{i, j=1}^{N} b_{i j} x_{j} \partial_{x_{i}} u(t, x)-\partial_{t} u(t, x)
$$

The linear stochastic differential equation in $\mathbb{R}^{N}$ associated to $K$ is the following:

$$
d Z_{t}=B Z_{t} d t+\sigma d W_{t}, \quad Z_{s}=z
$$

where $W$ is a standard $m$-dimensional Brownian motion, $B$ is a $N \times N$ constant matrix and $\sigma$ is the $N \times m$ constant matrix

$$
\sigma=\left(\begin{array}{c}
I_{m} \\
0
\end{array}\right)
$$

where $I_{m}$ denotes the identity matrix in $\mathbb{R}^{m}$. Then the solution of 10 is a Gaussian process with mean vector

$$
E\left[Z_{t}\right]=e^{(t-s) B} z
$$

and covariance matrix $\mathscr{C}_{0}(t-s)$ where

$$
\mathscr{C}_{0}(t)=\int_{0}^{t} e^{(t-\rho) B} \sigma \sigma^{*} e^{(t-\rho) B^{*}} d \rho, \quad t \geq 0
$$

Since $\sigma$ has dimension $N \times m$, the matrix $\mathscr{C}_{0}(t)$ is generally only positive semidefinite in $\mathbb{R}^{N}$, that is $Z_{t}$ possibly has degenerate multi-normal distribution. We recall the well-known Kalman condition from control theory provides an operative criterion for the positivity of $\mathscr{C}_{0}(t)$ : the matrix $\mathscr{C}_{0}(t)$ is positive definite for $t>0$ if and only if

$$
\operatorname{rank}\left[\sigma, B \sigma, B^{2} \sigma, \ldots, B^{N-1} \sigma\right]=N
$$

Then (12) ensures that $Z_{t}$ has a Gaussian transition density

$$
G(s, y ; t, x)=\frac{1}{\sqrt{(2 \pi)^{N} \operatorname{det} \mathscr{C}_{0}(t-s)}} \exp \left(-\frac{1}{2}\left\langle\mathscr{C}_{0}^{-1}(t-s)\left(x-e^{(t-s) B} y\right), x-e^{(t-s) B} y\right) .\right.
$$

Furthermore $G$ is the fundamental solution of the Kolmogorov PDE associated to (10). 
The fundamental solution under special assumptions has been found by Kolmogorov and Hörmander [65], but a systematic study of the the operator (9) has been done by Lanconelli and Polidoro in [78]. In particular they recognized that the hypoellipticity is equivalent to the following explicit expression of $B$, with respect to a suitable basis of $\mathbb{R}^{N}: B=\left(b_{i j}\right)_{i, j=1, \ldots, N}$ writes in the form

$$
B=\left(\begin{array}{ccccc}
* & * & \cdots & * & * \\
B_{1} & * & \cdots & * & * \\
0 & B_{2} & \cdots & * & * \\
\vdots & \vdots & \ddots & \vdots & \vdots \\
0 & 0 & \cdots & B_{r} & *
\end{array}\right)
$$

where each $B_{j}$ is a $p_{j} \times p_{j-1}$ matrix with rank $p_{j}$, with

$$
p_{0}=m \geq p_{1} \geq \cdots \geq p_{r} \geq 1, \quad \sum_{j=0}^{r} p_{j}=N,
$$

and the $*$-blocks are arbitrary. Let us explicitly recall that the stratification condition implies in a standard way that in canonical coordinates there is a dilation and a translation naturally associated to the vector fields.

Using the existence of the fundamental solution for the constant coefficient operator, from the parametrix method it follows:

Theorem 2.1. Assume that $\left(a_{i j}(t, x)\right)_{i, j=1, \ldots, m}$ is symmetric with Hölder continuous entries and satisfies (2) for some positive constant $\lambda$. Then the operator $L$ defiend in (8) has a fundamental solution $\Gamma$. Moreover, for any $T>0$ there exist some positive constants $c^{-}, c^{+}, \lambda^{-}, \lambda^{+}$such that

$$
\begin{aligned}
c^{-} \Gamma^{-}(t, x ; \tau, \xi) & \leq \Gamma(t, x ; \tau, \xi) \leq c^{+} \Gamma^{+}(t, x ; \tau, \xi), \\
\left|\partial_{x_{j}} \Gamma(t, x ; \tau, \xi)\right| & \leq \frac{c^{+}}{\sqrt{\tau-t}} \Gamma^{+}(t, x ; \tau, \xi),
\end{aligned}
$$

for any $(t, x),(\tau, \xi)$ with $0<\tau-t<T$. Here $\Gamma^{ \pm}$is the fundamental solution of $L$ in 9) with constant coefficients $a_{i j}^{+}=\lambda^{+} \delta_{i j}, a_{i j}^{-}=\lambda^{-} \delta_{i j}$.

We outline the proof of Theorem 2.1 given in Polidoro [96] and Di Francesco and Pascucci [51].

Sketch of the proof. For fixed $w \in \mathbb{R}^{1+N}$, we denote by $\Gamma_{w}(z ; \zeta)$ the fundamental solution of the model operator $L_{w}$, with constant coefficients evaluated at the point w

$$
L_{w} u:=\sum_{i, j=1}^{m} a_{i j}(w) X_{i} X_{j} u-X_{0} .
$$

Then we call parametrix the function

$$
Z(z ; \zeta)=\Gamma_{\zeta}(z ; \zeta)
$$


We remark that $Z$ is a good approximation of $\Gamma$ near $\zeta$ and the expression of $Z$ can be estimated explicitly. Then we suppose that the fundamental solution takes the form:

$$
\Gamma(z ; \zeta)=Z(z ; \zeta)+\int_{0}^{t} \int_{\mathbb{R}^{N}} Z(z ; w) G(w ; \zeta) d w .
$$

In order to find the unknown function $G$, we impose that $\Gamma$ is the solution to the equation $L \Gamma(\cdot ; \zeta)=0$ in $] 0,+\infty\left[\times \mathbb{R}^{N}\right.$ : we wish to point out one more time, to make this totally transparent, that the operator $L$ acts on the variable $z$ while the point $\zeta$ is fixed. Then formally we obtain

$$
\begin{aligned}
0=L \Gamma(z ; \zeta) & =L Z(z ; \zeta)+L \iint_{] 0, T\left[\times \mathbb{R}^{N}\right.} Z(z ; w) G(w ; \zeta) d w \\
& =L Z(z ; \zeta)+\iint_{] 0, T\left[\times \mathbb{R}^{N}\right.} L Z(z ; w) G(w ; \zeta) d w-G(z ; \zeta),
\end{aligned}
$$

hence

$$
G(z ; \zeta)=L Z(z ; \zeta)+\iint_{] 0, T\left[\times \mathbb{R}^{N}\right.} L Z(z ; w) G(w ; \zeta) d w .
$$

Therefore $G$ is a solution of an integral equation equivalent to a fixed-point problem that can be solved by the method of successive approximations:

$$
G(z ; \zeta)=\sum_{k=1}^{+\infty}(L Z)_{k}(z ; \zeta)
$$

where

$$
\begin{aligned}
(L Z)_{1}(z ; \zeta) & =L Z(z ; \zeta), \\
(L Z)_{k+1}(z ; \zeta) & =\iint_{] 0, T\left[\times \mathbb{R}^{N}\right.} L Z(z ; w)(L Z)_{k}(w ; \zeta) d w, \quad k \in \mathbb{N} .
\end{aligned}
$$

It is possible to prove that there exists $k_{0} \in \mathbb{N}$ such that, for all $T>0$ and $\zeta=(0, y) \in$ $\mathbb{R}^{1+N}$, the function $(L Z)_{k}(\cdot ; \zeta)$ is continuous and bounded for any $k \geq k_{0}$. Moreover the series

$$
\sum_{k=k_{0}}^{+\infty}(L Z)_{k}(\cdot ; \zeta)
$$

converges uniformly on the strip $] 0, T\left[\times \mathbb{R}^{N}\right.$. Furthermore, the function $G(\cdot, \zeta)$ defined by 19$]$ is a solution to the integral equation 18 in $] 0, T\left[\times \mathbb{R}^{N}\right.$ and $\Gamma$ in 17$]$ is a fundamental solution to $L$.

Remark 2.1. The method also gives some pointwise estimates of the fundamental solution and its derivatives. We refer to Corielli, Foschi and Pascucci [48] where the accuracy of the parametrix method is studied to obtain numerical approximations for financial problems. 
Remark 2.1. There exists a positive constant $M$ and, for every $T>0$, there exists $c=c(T)>0$ such that

$$
\frac{e^{-M d((t, x),(\tau, y))^{2} /(t-\tau)}}{c|B((t, x), \sqrt{t-\tau})|} \leq \Gamma_{A}((x, t),(y, \tau)) \leq \frac{c e^{-d((t, x),(\tau, y))^{2} / M(t-\tau)}}{|B((t, x), \sqrt{t-\tau})|}
$$

for any $(t, x),(\tau, y)$ with $0<\tau-t<T$, where $d$ is the distance defined by the vector fields. Gaussian estimates for a general equation like (11) have been obtained by [26], [37]

\subsection{Gaussian estimates for the fundamental solution of Heat operators}

An other particularly notable class of operators of type (1) is given by the heat operators

$$
L_{A}=\sum_{i, j=1}^{m} a_{i j}(t, x) X_{i} X_{j}-\partial_{t}
$$

For sum of squares of vector fields operators of the kind (1) with left invariant homogeneous vector fields on Lie groups, Gaussian bounds have been proved by Varopoulos (see [107] and references therein). In absence of a group structure, Gaussian bounds have been proved, on a compact manifold and for finite time, by Jerison-Sanchez-Calle [66], with an analytic approach and, on the whole $\mathbb{R}^{N+1}$, by Kusuoka-Stroock, (see [71] and references therein), using the Malliavin stochastic calculus.

In a long series of papers Bonfiglioli, Lanconelli, Uguzzoni [19], [20], [22], Bramanti, Brandolini, Lanconelli, Uguzzoni [28], proved new Gaussian bounds for the operator $L_{A}$ with Hölder continuous coefficients. In the first papers the vector fields were assumed to belong to a Carnot group. Then, in [28] the results are presented in the full generality of $C^{\infty}$ vector fields satisfying the Hörmander condition. In this last case, the operator $L_{A}$ is initially assumed defined only on a cylinder $\mathbb{R} \times \Omega$ for some bounded $\Omega$, but, in order to obtain asymptotic estimates, it is extended to the whole space $\mathbb{R}^{N+1}$, in such a way that, outside a compact spatial set, it coincides with the classical heat operator. Henceforth all our statements will be referred to this extended operator.

Theorem 2.2 (Gaussian bounds). There exists a positive constant $M$ and, for every $T>0$, there exists a positive constant $c=c(T)$ such that, for $0<t-\tau \leq T, x, \xi \in$ $\mathbb{R}^{N}$, the following estimates hold

$$
\frac{e^{-M d(x, \xi)^{2} /(t-\tau)}}{c|B(x, \sqrt{t-\tau})|} \leq \Gamma_{A}(t, x ; \tau, \xi) \leq \frac{c e^{-d(x, \xi)^{2} / M(t-\tau)}}{|B(x, \sqrt{t-\tau})|}
$$




$$
\begin{gathered}
\left|X_{i} \Gamma_{A}(t, \cdot ; \tau, \xi)(x)\right| \leq \frac{c e^{-d(x, \xi)^{2} / M(t-\tau)}}{(t-\tau)^{-1 / 2}|B(x, \sqrt{t-\tau})|^{-1}} \\
\left|X_{i} X_{j} \Gamma_{A}(t, \cdot ; \tau, \xi)(x)\right|+\left|\partial_{t} \Gamma_{A}(\cdot, x ; \tau, \xi)(t)\right| \leq \frac{c e^{-d(x, \xi)^{2} / M(t-\tau)}}{(t-\tau)|B(x, \sqrt{t-\tau})|}
\end{gathered}
$$

where $|B(x, r)|$ denotes the Lebesgue measure of the purely spatial $d$-CarnotCarathéodory ball in $\mathbb{R}^{N}$.

We explicitly note that this estimate is analogous to the estimate 20] for the Komogorov equation, but here the distance in $[0, T] \times \mathbb{R}^{N}$ splits in the sum of a purely spatial one and a purely temporal one. Hence in this case

$$
\frac{d_{2}((x, t),(\xi, \tau))^{2}}{M(t-\tau)} \leq \frac{d(x, \xi)^{2}}{M(t-\tau)}+C
$$

allowing to discard the temporal part of the distance in the estimate.

As a main step in the proof of these bounds, they first consider constant coefficients operators: the point here is to handle carefully the dependence on the matrix $A$ and obtain uniform estimates, in the ellipticity class of the matrix $A$. To prove these uniform bounds, in [19] the authors exploited direct methods and the previous results in [23], [24]. While in [28] the authors have followed as close as possible the techniques of [66], the main new difficulties being the following: first, they have to take into account the dependence on the matrix $A$, getting estimates depending on $A$ only through the number $\lambda$; second, the estimates have to be global in space, while in [66] they work on a compact manifold; third, they need estimates on the difference of the fundamental solutions of two operators which have no analogue in [66]. The procedure is technically involved, it makes use of the uniform estimates [19] on groups, and a crucial role is played by the Rothschild-Stein lifting theorem [98].

Once obtained the uniform estimates for the model operator with constant coefficients, one can apply the Levi parametrix method and establish existence and Gaussian bounds for the fundamental solution of the operators with variable Hölder continuous coefficients $a_{i j}$.

Theorem 2.3 (Existence of a fundamental solution). Under the above assumptions, there exists a global fundamental solution $\Gamma_{A}(t, x ; \tau, \xi)$ for $L_{A}$ in $\mathbb{R}^{N+1}$, with the properties listed below.

(i) $\Gamma_{A}$ is a continuous function away from the diagonal of $\mathbb{R}^{N+1} \times \mathbb{R}^{N+1} ; \Gamma_{A}(t, x ; \tau, \xi)=$ 0 for $t \leq \tau$. Moreover, for every fixed $\zeta \in \mathbb{R}^{N+1}, \Gamma_{A}(\cdot ; \zeta) \in C_{\text {loc }}^{2, \alpha}\left(\mathbb{R}^{N+1} \backslash\{\zeta\}\right)$, and we have

$$
L_{A}\left(\Gamma_{A}(\cdot ; \zeta)\right)=0 \quad \text { in } \mathbb{R}^{N+1} \backslash\{\zeta\} .
$$

(ii) For every $\psi \in C_{0}^{\infty}\left(\mathbb{R}^{N+1}\right)$, the function $w(z)=\int_{\mathbb{R}^{N+1}} \Gamma_{A}(z ; \zeta) \psi(\zeta) d \zeta$ belongs to the class $C_{\text {loc }}^{2, \alpha}\left(\mathbb{R}^{N+1}\right)$, and we have 


$$
L_{A} w=-\psi \quad \text { in } \mathbb{R}^{N+1} .
$$

(iii) Let $\mu \geq 0$ and $T_{2}>T_{1}$ be such that $\left(T_{2}-T_{1}\right) \mu$ is small enough. Then, for every $f \in C^{\bar{\beta}}\left(\left[T_{1}, T_{2}\right] \times \mathbb{R}^{N}\right)$ (where $\left.0<\beta \leq \alpha\right)$ and $g \in C\left(\mathbb{R}^{N}\right)$ satisfying the growth condition $|f(x, t)|,|g(x)| \leq c \exp \left(\mu d(x, 0)^{2}\right)$ for some constant $c>0$, the function

$$
u(x, t)=\int_{\mathbb{R}^{N}} \Gamma_{A}\left(t, x ; T_{1}, \xi\right) g(\xi) d \xi+\int_{\left[T_{1}, t\right] \times \mathbb{R}^{N}} \Gamma_{A}(t, x ; \tau, \xi) f(\tau, \xi) d \tau d \xi,
$$

$x \in \mathbb{R}^{N}, t \in\left(T_{1}, T_{2}\right]$, belongs to the class $C_{\text {loc }}^{2, \beta}\left(\left(T_{1}, T_{2}\right) \times \mathbb{R}^{N}\right) \cap C\left(\left[T_{1}, T_{2}\right] \times \mathbb{R}^{N}\right)$. Moreover, $u$ is a solution to the following Cauchy problem

$$
L_{A} u=-f \text { in }\left(T_{1}, T_{2}\right) \times \mathbb{R}^{N}, \quad u\left(\cdot, T_{1}\right)=g \text { in } \mathbb{R}^{N}
$$

The proof follows the same ideas of the analogous presented in the previous section. The parametrix function is built starting from the fundamental solution $\Gamma_{w}$ of the constant coefficient operator

$$
L_{w}=\sum_{i, j=1}^{m} a_{i j}(w)(t, x) X_{i} X_{j}-\partial_{t}
$$

\subsection{Fundamental solution of more general operators}

Operators in the form of sum of squares of Hörmander vector fields with drift

$$
L=\sum_{j=1}^{m} X_{j}^{2}-X_{0}
$$

write in the form (1) as $A$ is the $m \times m$ identity matrix. Kogoj and Lanconelli consider this kind of operators in in [67], under the assumption that every pair of points $(t, x)$ and $(\tau, \xi)$ with $t<\tau$ can be joined by a Lipschitz path $\gamma$ which solves almost everywhere the non-autonomous ODE

$$
\gamma^{\prime}(s)=\sum_{i=1}^{m} \beta_{i}(s) X_{i}(\gamma(s))+\beta_{0}(s) X_{0}(\gamma(s))
$$

with $\beta_{0}(s) \geq 0$ for almost every $s$. In the article [67], Kogoj and Lanconelli give a list of examples of operators satisfying [23), that include, among other examples, Kolmogorov operators, as well as heat operators with smoot coefficients. For this family of operators, they prove the existence of a fundamental solution $\Gamma(x, t, y, s)$, which is strictly positive in the set $\left\{(x, t) \in \mathbb{R}^{N+1} \mid t>s\right\}$, and Gaussian upper bounds for $\Gamma$. They also prove mean value formulas and Harnack inequalities for the positive solutions of $L u=0$. 
Based on the Harnack inequality proved in [67], and on the trhansaltion invariance, Pascucci and Polidoro prove in [92] sharp lower bounds for the fundamental solution of operators satisfying [23. More recently, the method used in [92] has been extended in [38] to the study of Hormander opeerators that do not satisfy (23). For instance, the operator $L=\partial_{x}^{2}+x^{2} \partial_{y}+\partial_{t}$ is considered in two space variables. The fundamental solution $\Gamma=\gamma(x, y, t, \xi, \eta, \tau)$ is supported in the set $\left\{(x, y, t) \in \mathbb{R}^{3} \mid t>\tau, y>\eta\right\}$, then no Gaussian estimates can be proved for this example. On the other hand, upper and lower bound have been proved by combining PDE methods and Malliavin calculus.

\section{Balls, Mean Value Formulas and Potential Theory}

An important aspect in the study of the geometric analysis associated with many of the PDEs discussed so far is the investigation of the underlying geometric properties naturally associated to these PDEs. Starting from the celebrated papers of Bony [25] and of Nagel, Stein, Wainger [88], it became clear that the properties of the exponential maps associated with the smooth vector fields play a crucial role in understanding the equivalence of the distances of the spaces. This notion has been weakened by Lanconelli and Morbidelli [73] to the notion of quasi exponential for Lipschitz continuous vector fields. Then they proved a Poincaré inequality under a ball box type assumption.

A complementary point of view, largely adopted by Lanconelli, is to choose the level sets of the fundamental solution as privileged class of balls for the operator. The main advantage of this perspective is that the level sets of the fundamental solution reflect the main properties of the operator, and in particular they give information on the directions of propagation, allowing to express in a natural and intrinsic way the Poincaré inequality and the Potential Theory results, properties which are classically expressed on the balls of the metric.

The first results extending the mean-value formulas from the classical Laplace setting to the parabolic one are due to Pini [93], Watson [108], Fabes and Garofalo [54], Lanconelli and Garofalo [60] and [61]. In the sub-Riemannian setting, a mean value theorem for sums of squares of vector fields has been proved by Hoh and Jacob [64], Citti, Garofalo and Lanconelli [41], while the formula for general Kolmogorov type operators of type (11) is due to Lanconelli and Pascucci [75]. It has been proved in [59], [33], [21] that there is a strict relation between the existence of representation formulas and the Poincaré inequality, which indeed are equivalent in some special cases.

The use of asymptotic average operators in the characterization of classical subharmonic functions has a long history, starting with the papers [9] of Blaschke, [94] of Privaloff, [6] of Beckenbach and Radó, up to the recent monograph [2] of Armitage and Gardiner. This direction of research has been deeply developed in the framework of Carnot groups by Bonfiglioli, Lanconelli and Uguzzoni in the monograph [21], and then by Bonfiglioli and Lanconelli, who obtained new results 
concerning with: Harnack-Liouville type theorems [11]; characterizations of subharmonicity [13] (see also the very recent paper [17]); average formulas and representation theorems [17]; formulas of Poisson \& Jensen type; maximum principles for open unbounded sets [12]; the Dirichlet problem with $L^{p}$ boundary data and the Hardy spaces associated with them [14]; the Eikonal equation and Bôcher-type theorems for the removal of singularities [15]; convexity properties of the mean-value formulas with respect to the radius [18]; Gauss-Koebe and Montel type normality results [5].

Finally we quote some results of Lanconelli [72], Abbondanza and Bonfiglioli [1], Kogoj, Lanconelli and Tralli [68], Kogoj and Tralli [69], who characterized the set on which a mean value formula can be proved as the level sets of the fundamental solution.

\subsection{Almost exponential maps and Poincaré inequality}

The most classical result on exponential mappings and properties of control balls is due to Nagel Stein Wainger [88].

An abstract version of these notions was provided in the paper [73] for a family $X_{1}, \ldots, X_{m}$ of Lipschitz continuous vector fields in $\mathbb{R}^{N}$. Indeed the authors introduced the notion of controllable almost exponential map and they showed that, if a suitable ball-box inclusion holds, then one can get a proof of a Poincaré-type inequality for the family $X_{1}, \ldots, X_{m}$. Next we will describe such result.

Definition 3.3. Let $\Omega \subset \mathbb{R}^{N}$ be an open set and let $Q$ be an open neighborhood of the origin in $\mathbb{R}^{N}$. We say that a $C^{1}$ map $E: \Omega \times Q \rightarrow \mathbb{R}^{N}$ is an almost exponential map if:

(i) the map $Q \ni h \mapsto E(x, h)$ is one-to-one for each $x \in \Omega$;

(ii) there is $C_{0}>1$ such that

$$
0<C_{0}^{-1}\left|\operatorname{det} \frac{\partial E}{\partial h}(x, 0)\right| \leq\left|\operatorname{det} \frac{\partial E}{\partial h}(x, h)\right| \leq C_{0}\left|\operatorname{det} \frac{\partial E}{\partial h}(x, 0)\right| \quad \text { for all } h \in Q .
$$

An almost exponential map is controllable if there are a hitting time $T>0$ and a control function $\gamma: \Omega \times Q \times[0, T] \rightarrow \mathbb{R}^{N}$ such that:

(iii) for each $(x, h) \in \Omega \times Q$, the path $t \mapsto \gamma(x, h, t)$ is subunit and it satisfies $\gamma(x, h, 0)=x$ and $\gamma(x, h, T(h))=E(x, h)$ for some $T(h) \leq T$;

(iv) For each $h \in Q$ and $t \in[0, T(h)]$, the map $x \mapsto \gamma(x, h, t)$ is one-to-one, of class $C^{1}$ and it satisfies for some $C_{0}>1$

$$
\left|\operatorname{det} \frac{\partial \gamma}{\partial x}(x, h, t)\right| \geq C_{0}^{-1} \quad \text { for all } x \in \Omega, h \in Q \text { and } t \in[0, T(h)] .
$$

Let us recall also the local doubling condition for the Lebesgue measure of control balls: for any compact $K$ there is $C_{D}$ and $r_{0}>0$ such that 


$$
\left|B\left(x_{0}, 2 r\right)\right| \leq C_{D}\left|B\left(x_{0}, r\right)\right| \quad \text { for all } x_{0} \in K \text { and } r \leq r_{0} .
$$

Now we are ready to give a condition wich ensures the Poincaré inequality.

Theorem 3.4 ([73], Theorem 2.1). Let $X_{1}, \ldots, X_{m}$ be a family of locally Lipschitzcontinuous vector fields in $\mathbb{R}^{N}$. Assume that the Lebesgue measure of CarnotCarathéodory balls is locally doubling. Let $K \subset \mathbb{R}^{N}$ be a compact set and let $B=B\left(x_{0}, r\right)$ be a ball such that $x_{0} \in K$ and $r \leq r_{0}$. Assume that for a suitable $C_{0}>0$ there are open sets $Q \supset\{0\}, \Omega \subset B$ and an almost exponential map $E: \Omega \times Q \rightarrow \mathbb{R}^{N}$ such that

1) $|\Omega|>C_{0}^{-1}|B|$;

2) The map $E$ is controllable with a control $\gamma$ having hitting time $T \leq C_{0} r$;

$3)$ we have the inclusion $B \subset E(x, Q)$ for each $x \in \Omega$.

Then, there is a constant $C_{1}$ depending on $C_{0}$ and $C_{D}$ such that

$$
\int_{B}\left|u(x)-u_{B}\right| d x \leq C_{1} r \int_{C_{1} B}|X u(x)| d x \quad \text { for all } u \in C^{1}\left(C_{1} B\right)
$$

Remark 3.4. Here $u_{B}$ denote the standard average on the ball $B$ :

$$
u_{B}=\frac{1}{|B|} \int_{B} u(y) d y .
$$

In the next section we will give a different definition of mean, to be used when the vector fields are associated to an operator.

For the proof we refer to the original paper [73]. Here we note that the method has been tested successfully in the case of Hörmander vector fields, with regular and non regular coefficients and on a class of vector fields introduced by Franchi and Lanconelli [57, 58] which have the form

$$
X_{1}=\partial_{x_{1}}, X_{2}=\lambda_{2}\left(x_{1}\right) \partial_{x_{2}}, \quad \ldots \quad, X_{n}=\lambda_{n}\left(x_{1}, \ldots, x_{n-1}\right) \partial_{x_{n}},
$$

where the functions $\lambda_{j}$ satisfy suitable assumptions. We refer to the discussion in [73, Section 3] for the proof that these vector fields fit in the framework of controllable almost exponential maps. Further results with minimal assumptions on the coefficients are due to [85], and with a slightly different technique to [30].

\subsection{Mean value formulas on level sets and Poincaré inequality}

We will present here mean value formulas, which have been constructed for different operators: in the subelliptic setting: by Citti, Garofalo and Lanconelli for sum of squares [41], Lanconelli and Pascucci for Kolmogorov-type operators [75]. The first corollary will be a Poincaré formula, to be compared with the one established in the previous section.

Let us consider a particular operator of type (1) 
The role of fundamental in Potential and Regularity Theory

$$
L_{A}:=\sum_{i, j=1}^{N} a_{i j} X_{i} X_{j}+X_{0}
$$

for a constant coefficient matrix $\left(a_{i j}\right)$ satisfying 2 . We will denote

$$
\Omega_{r}(x):=\left\{y \in \mathbb{R}^{N}: \Gamma(x, y)>1 / r\right\},
$$

so that $\partial \Omega_{r}(x)$ will be the level set of $\Gamma$.

Let $\Omega \subseteq \mathbb{R}^{N}$ be an open set and suppose $u$ is u.s.c. on $\Omega$. For every fixed $\alpha>0$, and every $x \in \mathbb{R}^{N}$ and $r>0$ such that $\overline{\Omega_{r}(x)} \subset \Omega$, we define the Surface Mean $m_{r}$ and the Solid Mean $M_{r}$ for a function $u$ :

$$
\begin{gathered}
m_{r}(u)(x)=\int_{\partial \Omega_{r}(x)} u(y) \frac{a_{i j}(y) X_{i} \Gamma_{x}(y) X_{j} \Gamma_{x}(y)}{\left|\nabla_{E} \Gamma_{x}(y)\right|} \mathrm{d} \sigma(y), \\
M_{r}^{\alpha}(u)(x)=\frac{\alpha+1}{r^{\alpha+1}} \int_{0}^{r} \rho^{\alpha} m_{\rho}(u)(x) \mathrm{d} \rho,
\end{gathered}
$$

where $\nabla_{E}$ denotes the Euclidean gradient, and $\Gamma_{x}(y)=\Gamma(x, y)$. Here $\sigma$ denotes the Hausdorff $(N-1)$-dimensional measure in $\mathbb{R}^{N}$. We also denote

$$
I_{r}(x)=\frac{\alpha+1}{r^{\alpha+1}} \int_{0}^{r} \rho^{\alpha}\left(\int_{\Omega_{\rho}(x)} a_{i j} X_{i} \Gamma(x, y) X_{j} u(y) \mathrm{d} y\right) \mathrm{d} \rho,
$$

The following theorem, for the special case $X_{0}=0$ has been proved in [41], A general formula has been established by [75] for Kolmogorov equations, which redudes to the following one, when $\operatorname{div} X_{0}=0$.

Theorem 3.5. Then, for every function u of class $C^{2}$ on an open set containing $\overline{\Omega_{r}(x)}$, we have the following mean value formulas:

$$
u(x)=m_{r}(u)(x)-\int_{\Omega_{r}(x)} a_{i j} X_{i} \Gamma(x, y) X_{j} u(y) \mathrm{d} y, \quad u(x)=M_{r}^{\alpha}(u)(x)-I_{r}(x)
$$

In [43] the authors remarked that the Poincare inequality can be obtained by means of the mean value formula for a very special class of vector fields, with minimal regularity of the coefficients in the same spirit of [30] and [85]. Precisely when $X_{0}=0$, and there exists a continuous function $\varphi$ such that

$$
X_{i}=\partial_{x_{i}}-x_{i+n} \partial_{x_{2 n}}, X_{n}=\partial_{x_{n}}+2 \varphi(x) \partial_{x_{2 n}}, X_{i+n}=\partial_{x_{i+n}}+x_{i} \partial_{x_{2 n}},
$$

$i=1, \ldots, n-1$. These vector fields satisfy the Hörmander condition, and $\varphi$ is continuous, so that there is a CC distance associated to these vector fields.

Theorem 3.6. Let $\Omega$ be an open set. Assume that the function $\varphi$ and $u$ are Lipschitz continous defined on $\Omega$ with respect to the CC distance associated to these vector fields. For every compact set $K \subset \Omega$ there exist positive constants $C_{1}, C_{2}$ with $C_{2}>1$ (depending continuosly on the Lipschitz constant of $\varphi$ ) such that if $\overline{\Omega_{C_{2} r}(\bar{x})} \subset K$, we have 


$$
\int_{\Omega_{r}(\bar{x})}\left|u(x)-u_{\Omega_{r}(\bar{x})}\right| d x \leq C_{1} r \int_{\Omega_{C_{2} r}(\bar{x})}|\nabla u|
$$

\subsection{L-subharmonicity and average operators}

As we shall see soon, mean value formulas naturally allow the characterizations of the $\mathscr{L}$-subharmonic functions, and the derivation of an in-depth Potential Theory for $\mathscr{L}$.

Let

$$
\mathscr{L}:=\sum_{i, j=1}^{N} \partial_{x_{i}}\left(a_{i, j}(x) \partial_{x_{j}}\right)=\operatorname{div}(A(x) \nabla)
$$

be a linear second order PDO in $\mathbb{R}^{N}$, in divergence form, with $C^{\infty}$ coefficients and such that the matrix $A(x):=\left(a_{i, j}(x)\right)_{i, j \leq N}$ is symmetric and nonnegative definite at any point $x=\left(x_{1}, \ldots, x_{N}\right) \in \mathbb{R}^{N}$. The operator $\mathscr{L}$ is (possibly) degenerate elliptic. However, we assume that $\mathscr{L}$ is not totally degenerate at every point. Precisely, we assume that the following condition holds: there exists $i \in\{1, \ldots, N\}$ such that $a_{i, i}>$ 0 on $\mathbb{R}^{N}$. This condition, together with $A(x) \geq 0$, implies the well-known Picone's Maximum Principle for $\mathscr{L}$.

A function $h$ will be said $\mathscr{L}$-harmonic in an open set $\Omega \subseteq \mathbb{R}^{N}$ if $h \in C^{2}(\Omega, \mathbb{R})$ and $\mathscr{L} h=0$ in $\Omega$. An upper semicontinuous function (u.s.c. function, for short) $u: \Omega \rightarrow[-\infty, \infty)$ will be called $\mathscr{L}$-subharmonic in $\Omega$ if:

1. the set $\Omega(u):=\{x \in \Omega \mid u(x)>-\infty\}$ contains at least one point of every (connected) component of $\Omega$, and

2. for every bounded open set $V \subset \bar{V} \subset \Omega$ and for every $\mathscr{L}$-harmonic function $h \in C^{2}(V, \mathbb{R}) \cap C(\bar{V}, \mathbb{R})$ such that $u \leq h$ on $\partial V$, one has $u \leq h$ in $V$.

We shall denote by $\mathscr{S}(\Omega)$ the family of the $\mathscr{L}$-subharmonic functions in $\Omega$.

It is well known that the subharmonic functions play crucial roles in Potential Theory of linear second order PDEs (just think about Perron's method for the Dirichlet problem) as well as in studying the notion of convexity in Euclidean and non-Euclidean settings.

Our main assumption on $\mathscr{L}$ is that it is $C^{\infty}$-hypoelliptic in every open subset of $\mathbb{R}^{N}$. We further assume that, in the spirit of the rest of the present paper, $\mathscr{L}$ admits a nonnegative global fundamental solution

$$
\mathbb{R}^{N} \times \mathbb{R}^{N} \backslash\{x=y\} \ni(x, y) \mapsto \Gamma(x, y) \in \mathbb{R},
$$

with pole at any point of the diagonal $\{x=y\}$ of $\mathbb{R}^{N}$ and vanishing at infinity.

We are then able to define suitable mean value operators on the level sets of $\Gamma$.

We explicitly remark that study of the integral operators related to general PDOs considered in this paper is complicated by the presence of non-trivial kernels. For instance, when $\mathscr{L}$ in 31 is a sub-Laplacian on a stratified Lie group $\mathbb{G}$, the kernels 
appearing in the relevant mean-integrals cannot be identically 1, unless $\mathbb{G}$ is the usual Euclidean group $\left(\mathbb{R}^{N},+\right)$, as it is proved in [15].

Definition 3.6 (Mean-Integral Operators). Let $x \in \mathbb{R}^{N}$ and let us consider the functions, defined for $y \neq x$,

$$
\Gamma_{x}(y):=\Gamma(x, y), \quad \mathscr{K}_{x}(y):=\frac{\left\langle A(y) \nabla \Gamma_{x}(y), \nabla \Gamma_{x}(y)\right\rangle}{\left|\nabla \Gamma_{x}(y)\right|} .
$$

We will call surface mean integral operator and solid mean integral operator, the two mean operators $m_{r}$ and $M_{r}$ defined in 26) and (27), respectively. Furthermore, for every $x \in \mathbb{R}^{N}$ and every $r>0$, we set

$$
\begin{aligned}
& q_{r}(x)=\int_{\Omega_{r}(x)}\left(\Gamma_{x}(y)-\frac{1}{r}\right) \mathrm{d} y, \quad Q_{r}(x)=\frac{\alpha+1}{r^{\alpha+1}} \int_{0}^{r} \rho^{\alpha} q_{\rho}(x) \mathrm{d} \rho, \\
& \omega_{r}(x)=\frac{1}{\alpha r^{\alpha+1}} \int_{\Omega_{r}(x)}\left(r^{\alpha}-\Gamma_{x}^{-\alpha}(y)\right) \mathrm{d} y .
\end{aligned}
$$

Remarkable mean-value formulas generalizing the classical Gauss-Green formulas for Laplace's operator and the ones in Theorem 3.5 hold true also in this more general setting:

Theorem 3.7 (Mean-Value Formulas for $\mathscr{L}$ ). Let $m_{r}, M_{r}^{\alpha}$ be the average operators in Definition 3.6. Let also $x \in \mathbb{R}^{N}$ and $r>0$.

Then, for every function u of class $C^{2}$ on an open set containing $\overline{\Omega_{r}(x)}$, we have the following $\mathscr{L}$-representation formulas:

$$
\begin{aligned}
& u(x)=m_{r}(u)(x)-\int_{\Omega_{r}(x)}\left(\Gamma(x, y)-\frac{1}{r}\right) \mathscr{L} u(y) \mathrm{d} y, \\
& u(x)=M_{r}^{\alpha}(u)(x)-\frac{\alpha+1}{r^{\alpha+1}} \int_{0}^{r} \rho^{\alpha}\left(\int_{\Omega_{\rho}(x)}\left(\Gamma(x, y)-\frac{1}{\rho}\right) \mathscr{L} u(y) \mathrm{d} y\right) \mathrm{d} \rho .
\end{aligned}
$$

We shall refer to (32) as the Surface Mean-Value Formula for $\mathscr{L}$, whereas (33) will be called the Solid Mean-Value Formula for $\mathscr{L}$.

Before stating our main theorem, we need two definitions. With the same notations as in the previous paragraph, an u.s.c. function $u$ defined on an open subset $\Omega$ of $\mathbb{R}^{N}$ will be called m-continuous in $\Omega$ if

$$
\lim _{r \rightarrow 0} m_{r}(u)(x)=u(x), \quad \text { for every } x \in \Omega .
$$

Analogously, $u$ is said to be $M^{\alpha}$-continuous in $\Omega$ if $\lim _{r \rightarrow 0} M_{r}^{\alpha}(u)(x)=u(x)$, for every $x \in \Omega$.

Finally, let $I \subseteq \mathbb{R}$ be an interval and suppose that $\varphi: I \rightarrow \mathbb{R}$ is a strictly monotone continuous function. We say that $f: I \rightarrow \mathbb{R}$ is $\varphi$-convex if

$$
f(r) \leq \frac{\varphi\left(r_{2}\right)-\varphi(r)}{\varphi\left(r_{2}\right)-\varphi\left(r_{1}\right)} f\left(r_{1}\right)+\frac{\varphi(r)-\varphi\left(r_{1}\right)}{\varphi\left(r_{2}\right)-\varphi\left(r_{1}\right)} f\left(r_{2}\right),
$$


for every $r_{1}, r, r_{2} \in I$ such that $r_{1}<r<r_{2}$.

We are ready to present our main result (see [17, 18]). This generalizes previous results in [13]; in the case of sub-Laplacians on Carnot groups, the paramount role of mean value operators is shown in [1, 5, 11, 12, 14, 15, 16]; see also the comprehensive monograph [21].

Theorem 3.8 (Characterizations of Subharmonicity). Suppose $\mathscr{L}$ satisfies the above axioms. Let $\Omega$ be an open subset of $\mathbb{R}^{N}$ and let $u: \Omega \rightarrow[-\infty, \infty)$ be an u.s.c. function such that $\Omega(u)=\{x: u(x)>-\infty\}$ contains at least one point of every component of $\Omega$.

Let $q_{r}, Q_{r}, \omega_{r}$ be as in Definition 3.6. Let also $R(x):=\sup \left\{r>0: \Omega_{r}(x) \subseteq \Omega\right\}$. Then, the following conditions are equivalent:

1. $u \in \mathscr{S}(\Omega)$ with respect to $\mathscr{L}$.

2. $u(x) \leq m_{r}(u)(x)$, for every $x \in \Omega$ and $r \in(0, R(x))$.

3. $u(x) \leq M_{r}^{\alpha}(u)(x)$, for every $x \in \Omega$ and $r \in(0, R(x))$.

4. It holds that

$$
\limsup _{r \rightarrow 0} \frac{m_{r}(u)(x)-u(x)}{q_{r}(x)} \geq 0, \quad \text { for every } x \in \Omega(u) .
$$

5. It holds that

$$
\limsup _{r \rightarrow 0} \frac{M_{r}^{\alpha}(u)(x)-u(x)}{Q_{r}(x)} \geq 0, \quad \text { for every } x \in \Omega(u) .
$$

6. $u$ is m-continuous in $\Omega$, and $r \mapsto m_{r}(u)(x)$ is monotone increasing on $(0, R(x))$, for every $x \in \Omega$.

7. u is $M^{\alpha}$-continuous in $\Omega$, and $r \mapsto M_{r}^{\alpha}(u)(x)$ is monotone increasing on $(0, R(x))$, for every $x \in \Omega$.

8. $u$ is m-continuous in $\Omega$, and

$$
M_{r}^{\alpha}(u)(x) \leq m_{r}(u)(x),
$$

for every $x \in \Omega$ and every $r \in(0, R(x))$.

9. $u$ is m-continuous in $\Omega$, and

$$
\liminf _{r \rightarrow 0} \frac{m_{r}(u)(x)-M_{r}^{\alpha}(u)(x)}{\omega_{r}(x)} \geq 0, \quad \text { for every } x \in \Omega(u) .
$$

10. $u$ is $M^{\alpha}$-continuous in $\Omega, u \in L_{\mathrm{loc}}^{1}(\Omega)$ and $\mathscr{L} u \geq 0$ in the weak sense of distributions.

11. $u$ is $m$-continuous and the map $r \mapsto m_{r}(u)(x)$ is $\frac{1}{r}$-convex on $(0, R(x))$, for every $x \in \Omega$ (or, equivalently, for every $x \in \Omega(u)$ );

12. $u$ is $M^{\alpha}$-continuous and, for every $x \in \Omega$ (or, equivalently, for every $x \in \Omega(u)$ ), the map $r \mapsto M_{r}^{\alpha}(u)(x)$ is $\frac{1}{r^{\alpha+1}}$-convex on $(0, R(x))$, for some (or for every) $\alpha>$ 0 ; 
Furthermore, if $u \in \mathscr{S}\left(\mathbb{R}^{N}\right)$ we have the following results:

13. the functions $x \mapsto m_{r}(u)(x), M_{r}^{\alpha}(u)(x)$ are $\mathscr{L}$-subharmonic in $\mathbb{R}^{N}$, finite valued and continuous.

14. Let $\mu_{u}$ be the $\mathscr{L}$-Riesz measure of $u$; the maps $r \mapsto m_{r}(u)(x)$ and $r \mapsto M_{r}^{\alpha}(u)(x)$ can be prolonged with continuity up to $r=0$ if and only if $x \in \Omega(u)$.

Furthermore, for every $x \in \Omega$ and $r \in(0, R(x))$, one has the following representation formulas (of Poisson-Jensen type):

$$
\begin{aligned}
u(x) & =m_{r}(u)(x)-\int_{0}^{r} \frac{\mu_{u}\left(\Omega_{\rho}(x)\right)}{\rho^{2}} \mathrm{~d} \rho \\
& =m_{r}(u)(x)-\int_{\Omega_{r}(x)}\left(\Gamma(x, y)-\frac{1}{r}\right) \mathrm{d} \mu_{u}(y), \\
u(x) & =M_{r}^{\alpha}(u)(x)-\int_{0}^{r} \frac{\alpha+1}{\rho^{\alpha+2}}\left(\int_{\Omega_{\rho}(x)}\left(f_{\alpha}(\rho)-f_{\alpha}\left(\frac{1}{\Gamma(x, y)}\right)\right) \mathrm{d} \mu_{u}(y)\right) \mathrm{d} \rho \\
& =M_{r}^{\alpha}(u)(x)-\frac{\alpha+1}{r^{\alpha+1}} \int_{0}^{r} \rho^{\alpha}\left(\int_{\Omega_{\rho}(x)}\left(\Gamma(x, y)-\frac{1}{\rho}\right) \mathrm{d} \mu_{u}(y)\right) \mathrm{d} \rho .
\end{aligned}
$$

When $x \notin \Omega(u)$, all the sides of these formulas are $-\infty$, and this happens if and only if $\mu_{u}(\{x\})>0$.

The equivalences (1)-to-(9) do not require the hypoellipticity of $\mathscr{L}$, which is only used in (10)-to-(14) (requiring Riesz-type representation results).

We observe that Theorem 3.8 provides new insight on the Potential Theory for operators in divergence form, which are not necessarily in the form of Hörmander sums of squares, nor left invariant on some Lie group (see [17, 18]).

Finally we mention some results of Lanconelli [72], Abbondanza and Bonfiglioli [1], Kogoj, Lanconelli and Tralli [68], Kogoj and Tralli [69]: in these papers, it is proved, for several classes of PDOs, that the sets on which a mean value formula can be obtained are precisely the level sets of the fundamental solution. For instance the inverse mean value theorem for $\mathscr{L}$ states the following: let $\mathscr{K}_{0}(y)$ be as in Definition 3.6 and let us set $\mathrm{d} v(y):=\mathscr{K}_{0}(y) \mathrm{d} y$; let $D$ be a bounded open neighborhood of 0 such that

$$
u(0)=\frac{1}{v(D)} \int_{D} u(y) \mathrm{d} v(y),
$$

for every $u$ which is $\mathscr{L}$-harmonic and $v$-integrable on $D$. Then, necessarily, $D=$ $\Omega_{r}(0)$ for some $r>0$. More precisely, it suffices to suppose that 34 holds for the family of the $\mathscr{L}$-harmonic functions on $D$ of the form $D \ni y \mapsto \Gamma(y, x)$, for $x \notin D$.

\section{Global Regularity Results}

Interior Schauder and $L^{p}$ estimates can be obtained as a direct consequence of existence of the fundamamental solution. A much more delicate problem is the problem 
of global regularity results, namely regularity on the whole space, or regularity at the boundary.

We provide here a couple of results, obtained using potential theory and existence of the fundamental solution, proved in the previous section.

\subsection{A first regularity result at the boundary}

An essential play in study of the existence and regularity theory of the equation $L u=f$ where $L$ is the operator in (8) is the derivation of the Schauder estimates in terms of weighted interior norms. Such apriori estimates allow to extends the results of potential theory to the class of $L$ having Hölder continuous coefficients and to establish the solvability of the Dirichlet problem in the generalized sense. For continuous boundary values and a suitably wide class of bounded open set the proof of solvability of the Dirichlet problem can be achieved entirely with interior estimates.

Interior Schauder's estimates for the Kolmogorov operator (4) are proved in Shatyro [99], for the operator (8) in the homogeneous case by Manfredini in [79] and in the non homogeneous case in Di Francesco and Polidoro [52]. In Lunardi [77] global estimates with respect to the spatial variable are proved for operator (8) with constant coefficients $a_{i j}$.

We denote by $C_{d}^{\alpha}(\Omega)$ the space of the Hölder continuous function whose norms $|\cdot|_{\alpha, d ; \Omega}$ are weighted by the distance to the boundary of the bounded open set $\Omega$. Schauder's type estimate can be proved using classical arguments, based on a representation formula for the second derivatives of smooth functions in terms of the fundamental solution of the operator and on its bounds in Theorem 2.1.

Theorem 4.9. (Schauder interior estimates). Let $\Omega$ be a bounded open set, $f \in$ $C_{d}^{\alpha}(\Omega)$, and let $u$ be a bounded function belonging to $C_{l o c}^{2+\alpha}(\Omega)$ such that $L u=f$ in $\Omega$. Then $u \in C_{d}^{2+\alpha}(\Omega)$ and there exists a positive constant $c$, independent of $u$, such that

$$
|u|_{2+\alpha, d ; \Omega} \leq c\left(\sup _{\Omega}|u|+\left|d^{2} f\right|_{\alpha, d ; \Omega}\right) .
$$

Here $\left|d^{2} f\right|_{\alpha, d ; \Omega}$ denotes the following norm :

$$
\left|d^{2} f\right|_{\alpha, d ; \Omega}=\sup _{z \in \Omega} d_{z}^{2}|f(z)|+\sup _{z, \zeta \in \Omega, z \neq \zeta} d_{z, \zeta}^{2+\alpha} \frac{|f(z)-f(\zeta)|}{d(z, \zeta)^{\alpha}}
$$

where $d_{z}=\inf _{w \in \Omega} d(z, w)$ and $d_{z, \zeta}=\min \left\{d_{z}, d_{\zeta}\right\}$.

And 


$$
\begin{aligned}
|u|_{2+\alpha, d ; \Omega}= & \sup _{z \in \Omega}|u(z)|+\sum_{i=1}^{m} \sup _{z \in \Omega} d_{z}\left|\partial_{x_{i}} u(z)\right|+\sup _{z, \zeta \in \Omega, z \neq \zeta} d_{z, \zeta}^{2+\alpha} \frac{|u(z)-u(\zeta)|}{d(z, \zeta)^{\alpha}} \\
& +\sum_{i=1}^{m} \sup _{z, \zeta \in \Omega, z \neq \zeta} d_{z, \zeta}^{2+\alpha} \frac{\left|\partial_{x_{i}} u(z)-\partial_{x_{i}} u(\zeta)\right|}{d(z, \zeta)^{\alpha}}+\left|d^{2} X_{0} u\right|_{\alpha, d ; \Omega}+\sum_{i, j=1}^{m}\left|d^{2} \partial_{x_{i} x_{j}}^{2} u\right|_{\alpha, d ; \Omega} .
\end{aligned}
$$

Using Schauder apriori estimates we can extend potential theory to the operator $L$ with Hölder continuous coefficients. In fact, $L$ endows $\mathbb{R}^{N+1}$ with a structure of $\beta$-harmonic space (according to the classical definition in [47]). Precisely, if $U$ is a bounded subset of $\mathbb{R}^{N+1}$ the space $\left(U, H^{L}\right)$ of $L$-harmonic $C_{l o c}^{2+\alpha}(U)$ functions satisfies the axiom of positivity and separation, the Doob convergence property and finally the property of resolutivity. In particular the last axiom requires that there exists a basis (for the Euclidean topology) of bounded open set $V$ called $H^{L}$-regular set such that the Dirichlet problem

$$
\left\{\begin{array}{l}
L u=0 \quad \text { in } V \\
u=\varphi \quad \text { in } \partial V, \varphi \in C(\partial V)
\end{array}\right.
$$

is univocally solvable. We cannot expect that the parabolic cylinders are $H^{L}$-regular set. A geometric condition on $\partial V$ ensuring the solvability of 36 is a generalization of the Poincaré exterior ball condition. Precisely, we assume that for every $\left(x_{0}, t_{0}\right) \in \partial V$ there exists a $L$-non-characteristic outer normal $v \in \mathbb{R}^{N+1}$ such that $B_{\text {eucl }}\left(\left(x_{0}, t_{0}\right)+v,|v|\right) \subset \mathbb{R}^{N+1} \backslash V$ and

$$
\sum_{i, j=1}^{m} a_{i j}\left(x_{0}, t_{0}\right)\left\langle v, X_{i}\right\rangle\left\langle v, X_{j}\right\rangle>0
$$

The construction of a basis of $H^{L}$-regular sets is proved using an argument due a Bony [25] and the method of continuity.

The general potential theory ensures the existence of a generalized solution in the sense of Perron-Wiener-Brelot-Bauer of the Dirichlet problem in an arbitrary bounded open set $\Omega$. This solution assumes the boundary data at every $L$-regular point. A point $\left(x_{0}, t_{0}\right)$ is $L$-regular if there exists a local barrier at $\left(x_{0}, t_{0}\right)$.

Theorem 4.10. [79] (Existence of a generalized solution). Let $\Omega$ be a bounded open set, $f \in C^{\alpha}(\Omega)$ and $\varphi \in C(\partial \Omega)$. Then, there exists a solution $u \in C_{l o c}^{2+\alpha}(\Omega)$ of $L u=f$ in $\Omega$ such that $\lim _{(x, t) \rightarrow\left(x_{0}, t_{0}\right)} u(z)=\varphi\left(x_{0}, t_{0}\right)$ for every L-regular point $\left(x_{0}, t_{0}\right) \in \partial \Omega$.

Geometric properties of the boundary determine the continuous assumption of boundary values. In the paper [79] the author introduce an exterior cone type condition which extends the classical Zaremba criteria for the regularity of the boundary points and a boundary condition for the Kolmogorov operator in $\mathbb{R}^{3}$ proved in [89]. Besides, a geometric condition ensures the regularity for the $L$-characteristic boundary point, when the Fichera function $X_{0} v\left(x_{0}, t_{0}\right)$ is positive definite.

Related results on the regularity of boundary points for the Dirichlet problem are also proved in [76], [105], [106], [104]. 


\subsection{A global regularity result in $L^{p}$ Spaces}

We conclude this section with $L^{p}$-regularity results on the whole space for degenerate Ornstein-Uhlenbeck operators obtained by Bramanti, Cupini, Lanconelli, Priola in [31] (constant coefficients) and [32] (variable coefficients).

The class of operators considered in [31] is

$$
\mathscr{A}=\sum_{i, j=1}^{p_{0}} a_{i j} X_{i} X_{j}+X_{0}
$$

where $1 \leq p_{0} \leq N, \quad X_{i}=\partial_{i}$ and $X_{0}=\sum_{i, j=1}^{N} b_{i j} x_{i} \partial_{x_{j}}$. Here $A=\left(a_{i j}\right)_{1 \leq i, j \leq p_{0}}$ and $B=\left(b_{i j}\right)_{1 \leq i, j \leq N}$ are constant coefficient matrices. Moreover, $B$ has the structure described in (14) and $A$ satisfies the ellipticity assumption (2), with $p_{0}$ in place of $m$.

The evolution operator corresponding to $\mathscr{A}$, that is

$$
L_{A}=\mathscr{A}-\partial_{t}
$$

is a Kolmogorov-Fokker-Planck ultraparabolic operator, studied in Section 2.1. For this operator, $L^{p}$ global estimates on the strip $S=\mathbb{R}^{N} \times[-1,1]$ have been proved in [31].

Theorem 4.11. For every $p \in(1, \infty)$ there exists a constant $c>0$ such that

$$
\left\|\partial_{x_{i} x_{j}}^{2} u\right\|_{L^{p}(S)} \leq c\|L u\|_{L^{p}(S)} \text { for } i, j=1,2, \ldots, p_{0}
$$

for every $u \in C_{0}^{\infty}(S)$. The constant $c$ depends on $p, N, p_{0}$, the matrix $B$ and the number $\lambda$ in (2).

As a by-product of the above result, global $L^{p}\left(\mathbb{R}^{N}\right)$ estimates are deduced for the operator $\mathscr{A}$.

Theorem 4.12. For every $p \in(1, \infty)$ there exists a constant $c>0$, such that for every $u \in C_{0}^{\infty}\left(\mathbb{R}^{N}\right)$ one has:

$$
\left\|\partial_{x_{i} x_{j}}^{2} u\right\|_{L^{p}\left(\mathbb{R}^{N}\right)} \leq c\left\{\|\mathscr{A} u\|_{L^{p}\left(\mathbb{R}^{N}\right)}+\|u\|_{L^{p}\left(\mathbb{R}^{N}\right)}\right\} \text { for } i, j=1,2, \ldots, p_{0} .
$$

We point out that the estimates of [98] for these type of operators only allow to get local estimates in $L^{p}$, while the results presented here are global.

The same authors prove in [32] similar estimates in the case of variable coefficients $a_{i j}$, entries of the matrix $A$. Precisely, if $a_{i j}$ are uniformly continuous and bounded functions in $\mathbb{R}^{N}$, estimates analogous to (37) (with $S=\mathbb{R}^{N} \times[-T, T]$, for some $T>0$ ) and (38) still hold true. The proofs of the results in [32] rely on a freezing argument, that allows to exploit results and techniques contained in [31], and useful estimates proved in [78] and [52]. 
Let us describe now the general strategy of the proof of Theorem 4.11 , as well as the main difficulties.

Since $B$ has the structure described in [14], with the $*$-blocks possibly not null, the operator $L$ is left invariant with respect to a suitable Lie group of translations, but, in general, is not homogeneous. A basic idea is that of linking the properties of $L$ to those of another operator of the same kind, which not only is left translation invariant, but is also homogeneous of degree 2 with respect to a family of dilations. Such an operator $L_{0}$ always exists under our assumptions by [78], and has been called "the principal part" of $L$. Note that the operator $L_{0}$ fits the assumptions of Folland's theory [55]. The authors exploit the fact that, by results proved in [52], the operator $L$ has a fundamental solution $\Gamma$ with some good properties. First of all, $\Gamma$ is translation invariant and has a fast decay at infinity, in space; this allows to reduce the desired $L^{p}$ estimates to estimates of a singular integral operator whose kernel vanishes far off the pole. Second, this singular kernel, which has the form $\eta \cdot \partial_{x_{i} x_{j}}^{2} \Gamma$ where $\eta$ is a radial cutoff function, satisfies "standard estimates" (in the language of singular integrals theory) with respect to a suitable "local quasisymmetric quasidistance" $d$, which is a key geometrical object in the paper under consideration. Namely,

$$
d(z, \zeta)=\left\|\zeta^{-1} \circ z\right\|
$$

where $\zeta^{-1} \circ z$ is the Lie group operation related to the operator $L$, while $\|\cdot\|$ is a homogeneous norm related to the principal part operator $L_{0}$ (recall that $L$ does not have an associated family of dilations, and therefore does not have a natural homogeneous norm). This "hybrid" quasidistance does not fulfill enough good properties in order to apply the standard theory of "singular integrals in spaces of homogeneous type" (in the sense of Coifman-Weiss [46]). Hence, an ad hoc theory of singular integrals in nonhomogeneous spaces (see [27]) and a nontrivial covering argument are applied to get the desired $L^{p}$ bound.

\section{Non linear curvature equations}

We conclude this review studying non linear PDE's. The standard prototype of non linear equations have always been minimal surfaces and curvature equations. Also in the setting of CR manifolds and subriemannian spaces, curvature equations can be chosen as the prototype of non linear equations. These equations describe the curvature or the evolution of a graph, with respect to vector fields, (or a metric) dependent on the graph itself. This is why curvature equation in this setting can be expressed in the form (1), where the coefficients $\sigma_{i j}=\sigma_{i j}\left(u, X_{i} u\right)$ of the vector fields depend on the solution or its intrinsic derivatives. Equations of this type naturally arise while studying curvature equations in CR manifolds, called Levi Equation ([39], [84]), Monge-Ampere equation ([102, 97]) or minimal graphs in the Heisenberg group (see for instance [34], [50], [86]), as well as in mathematical finance ([44, 53] ). 
Here we will focus in particular on Levi equations, for which much of the technique has been developed.

\subsection{Regularity results for the Levi equations}

The Levi curvatures of a graph is the formal complex analogous of the curvature operator in $\mathbb{R}^{N}$. Namely it is the determinant of Levi form of a real hypersurface in $\mathbb{C}^{n+1}$ (or elementary symmetric functions of it). We can always assume that the surface $M$ is the graph of a $C^{2}$ function $u: \Omega \rightarrow \mathbb{R}$, where $\Omega \subseteq \mathbb{R}^{2 n+1}$ is open. We identify $\mathbb{R}^{2 n+1} \times \mathbb{R}$ with $\mathbb{C}^{n+1}$, and denote

$$
z=(x, y)=\left(z_{1}, \ldots, z_{n}, z_{n+1}\right), z_{j}=x_{2 j-1}+i x_{2 j}, 1 \leq j \leq n, z_{n+1}=x_{2 n+1}+i y .
$$

We let

$$
\gamma(u)=\{(x, y) \in \Omega \times \mathbb{R}: y=u(x)\} \equiv \text { graph of } u .
$$

Calling $f(x, y)=y-u(x)$, the Levi form associated with $f$ at the point $p=(x, u(x))$ is the following Hermitian form:

$$
L_{p}(u, \zeta)=\sum_{j, k=1}^{n+1} f_{j, \bar{k}}(p) \zeta_{j} \bar{\zeta}_{k}, \quad \zeta \in T_{p}^{\mathbb{C}}(\gamma(u))
$$

where $T_{p}^{\mathbb{C}} \gamma(u)$ denotes the complex tangent space to the graph of $u$. If we denote by $h$ the complexified second fundamental form, it turns our that $h_{p}(\zeta, \bar{\zeta})=$ $\frac{1}{|\partial f(p)|} L_{p}(u, \zeta)$ for all $\zeta \in T_{p}^{\mathbb{C}}(\gamma(u))$. Let $\lambda_{1}(p), \ldots, \lambda_{n}(p)$ be the eigenvalues of $h$. For $1 \leq m \leq n, \sigma^{(m)}$ denotes the $m$-th elementary symmetric function and

$$
K_{p}^{(m)}(\partial D):=\frac{1}{\left(\begin{array}{c}
n \\
m
\end{array}\right)} \sigma^{(m)}\left(\lambda_{1}, \ldots, \lambda_{n}\right),
$$

we define the $m$-th Levi curvature operator as

$$
\mathscr{L}^{(m)}(u)(x):=K_{p}^{(m)}(\gamma(u)), \quad x \in \Omega,
$$

(see the papers by Bedford and Gaveau [7], by Tomassini [103], and by Lanconelli Montanari [84]).

The Levi form has been introduced by E.E. Levi and used by Oka, Bremmerman and Norgouet in order to characterize domains of holomorphy. The first existence results were obtained in the Levi flat case, i.e. null Levi form, by Bedford and Gaveau [7] and by Bedford and Klingenberg [8]. They used a purely geometric approach, which does not work in the non Levi flat case. Slodkowski and Tomassini in [100] introduced a PDE's approach in studying boundary value problems for the prescribed Levi curvature equation with curvature different from zero at any point 
and proved $L^{\infty}$ a-priori bound for the gradient. However the degeneracy of the equation did not allow the mentioned authors to obtain internal regularity with standard instruments. Almost ten years later the work by Slodkowski and Tomassini, in [39] G. Citti recasted the problem in dimension $n=1$ in the set of sum of squares of vector fields. Precisely, choosing the coefficients of the vector fields $X_{i}=\sigma_{i j} \partial_{x_{j}}$ of (1) as $\sigma_{i j}=\delta_{i j}$ for $i=1,2$,

$$
\sigma_{13}(D u)=\frac{u_{x_{2}}-u_{x_{1}} u_{x_{3}}}{1+u_{x_{3}}^{2}}, \quad \sigma_{23}(D u)=-\frac{u_{x_{1}}+u_{x_{2}} u_{x_{3}}}{1+u_{x_{3}}^{2}}
$$

and the Levi Curvature operator for $n=1$ can be expressed as

$$
\mathscr{L}^{(1)} u=\left(X_{1}^{2} u+X_{2}^{2} u\right)\left(1+u_{x_{3}}^{2}\right) \text { and }\left[X_{1}, X_{2}\right]=-\frac{\mathscr{L}^{(1)} u}{1+u_{x_{3}}^{2}} \partial_{x_{3}} .
$$

This representation tells us that, while prescribing the curvature, we can control the rank of the Lie algebra generated by the vector fields, allowing to apply to the equation the theory of subriemannian operators. E. Lanconelli and A. Montanari studied the problem in full generality (see [84]) proving that $\mathscr{L}^{(m)}$ can be written as follows:

$$
\mathscr{L}^{(m)}(u)(x)=\sum_{j, k=1}^{2 n} a_{j, k} Z_{j} Z_{k}(u), \quad u \in C^{2}(\Omega, \mathbb{R}), \quad \Omega \subset \mathbb{R}^{2 n+1}
$$

where

- $Z_{j}=\partial_{x_{j}}+a_{j} \partial_{x_{2 n+1}}, \quad a_{j}=a_{j}(D u), j=1, \cdots, 2 n$

- $\left(a_{j, k}\right)_{j, k=1, \ldots, 2 n}$ is symmetric and $a_{j, k}=a_{j, k}\left(D u, D^{2} u\right)$.

Then, if $j \neq k$, we have : $Z_{j, k}:=\left[Z_{j}, Z_{k}\right]=q_{j, k} \partial_{x_{2 n+1}}$. When computed on $m$-strictly pseudoconvex functions, i.e., on functions satisfying $\mathscr{L}^{(k)}(u)(x)>0$ for every $x \in \Omega$ and $1 \leq k \leq m$, the operator $\mathscr{L}^{(m)}$ displays a subelliptic property. Precisely:

- for every $x \in \Omega, q_{j, k}(x) \neq 0$ for suitable $j, k$

- the matrix $\left(a_{j, k}(x)\right)_{j, k=1, \ldots, 2 n}$ is strictly positive definite at any point $x \in \Omega$.

Therefore, if $u$ is $m$-strictly pseudoconvex, $\mathscr{L}^{(m)}$ is elliptic only along the $2 n$ linearly independent directions $Z_{j}=\partial_{x_{j}}+a_{j} \partial_{x_{2 n+1}} \equiv e_{j}+a_{j} e_{n+1}, j=1, \ldots, 2 n$, and the missing ellipticity direction $e_{2 n+1}$ is recovered by commutation. This commutation property can be restated as follows:

$$
\operatorname{dim}\left(\operatorname{span}\left\{Z_{j}(x), Z_{j, k}(x): j, k=1, \ldots, 2 n\right\}\right)=2 n+1, \quad \text { for every } x \in \Omega .
$$

We would also like to stress that $\mathscr{L}^{(m)}$ is a PDO in $\mathbb{R}^{2 n+1}$, which is fully nonlinear if $n>1$. 
From the subelliptic properties of $\mathscr{L}^{(m)}$ several crucial results follow. Here we only mention a Strong Comparison Principle and a regularity result.

Theorem 5.13 (STRONG COMPARISON PRINCIPLE). Let $u, v: \Omega \rightarrow \mathbb{R}$, where $\Omega \subseteq$ $\mathbb{R}^{2 n+1}$ is open and connected. Assume $u$ and $v$ strictly m-pseudoconvex and

(i) $u \leq v$ in $\Omega, u\left(x_{0}\right)=v\left(x_{0}\right)$ at $x_{0} \in \Omega$

(ii) $\mathscr{L}^{(m)}(u) \geq \mathscr{L}^{(m)}(v)$ in $\Omega$.

Then $u=v$ in $\Omega$ (see [39] for $n=1,[84]$ for the general case).

Theorem 5.14 (SMOOTHNESS OF CLASSICAL SOLUTIONS).

Let $u \in C^{2, \alpha}(\Omega)$ be a strictly m-pseudoconvex solution to the K-prescribed Levi curvature equation

$$
\mathscr{L}^{(m)}(u)=K(\cdot, u) \quad \text { in } \Omega .
$$

If $K$ is strictly positive and $C^{\infty}$ in its domain, then $u \in C^{\infty}(\Omega)$ (see [40] for $n=1$, [83] for $1 \leq m$ and [81] for the general case $1 \leq m \leq n$ ).

Strategy of the proof for $\mathbf{n}=\mathbf{1}$ In the low dimensional case, the proof of regularity is based on an ad hoc approximation method, similar to the parametrix method (see Section 2). The difficult here is the fact that the approximation has to be applied to the vector fields, not to the metric of the space. Following [98], the approximating vector fields $X_{i, w}$ of $X_{i}$ are obtained via Taylor approximation. The additional difficulty here is due to the fact that a function differentiable in the direction of the vectors $X_{i}$ will not necessarly be differentiable in the direction $X_{i, w}$. We explicitly note that not even the more recent results of Bramanti Brandolini Manfredini Pedroni [29] could allow to obtain the result. On the contrary a completely new approach to singular integrals has been introduced in order to deal with these non linear vector fields.

Strategy of the proof in higher dimension Since the prescribed Levi curvature equations present formal similarities with the real and complex MongeAmpère equations, which are elliptic PDE's if evaluated on strictly convex and plurisubharmonic functions, respectively, we would like to briefly recall how the smoothness follows from the classical Schauder theory for the real Monge-Ampère equation. The real Monge-Ampère equation in a domain $\Omega \subset \mathbb{R}^{n}$ is of the form $\operatorname{det}\left(D^{2} u\right)=f(x, u, D u)$. If $u \in C^{2, \alpha}(\Omega)$ is a strictly convex solution to this equation, then the linearized operator $L($ at $\mathrm{u})$ is elliptic with $C^{\alpha}$ coefficients, and $D u$ satisfies a linear uniformly elliptic equation of the type $L(D u)=F \in C^{\alpha}(\Omega)$. By the classical Schauder theory, $D u \in C^{2, \alpha}(\Omega)$. Repeating this argument one proves $u \in C^{\infty}(\Omega)$. In our case it is not possible to argue in the same way, because the Levi curvature equations are not elliptic at any point, also when restricted to the class of strictly pseudoconvex functions. However, in [82] Montanari proved interior Schauder-type estimates for solutions of $H v=f$ with $H$ a linear second order subelliptic operator of the type $H=\sum_{m, j=1}^{n} h_{m j} Z_{m} Z_{j}$ with Hölder continuous coefficients and with $Z_{j}$ first order partial differential operator with $C^{1, \alpha}$ coefficients. This result is obtained by a non standard freezing method and on the lifting argument by Rothshild and Stein. The study of the operator $H$ is reduced to the analysis of a family of left invariant operators on a free nilpotent Lie group, whose fundamental solutions are 
used a parametrix of the operator $H$, and provides an explicit representation formula for solutions of the linear equation $H v=f$. Once this is established, the strategy to handle the prescribed $m$-th Levi curvature equation in higher dimension is to apply the a priori estimates in [82] to first order Euclidean difference quotients of a strictly $m$-pseudoconvex solution $u$, in order to prove that the function $D u$ has Hölder continuous second order horizontal derivatives. The smoothness result is then obtain by a bootstrap argument.

\subsection{A negative regularity result}

We want to stress that, in dimension $n>1$, the classical $C^{2, \alpha}$ solvability of the Dirichlet problem for the $K$-prescribed Levi curvature equations is still a widely open problem. Even though it is possible to give a definition of Lipschitz continuos viscosity solutions (we refer [83]), these solutions are not expected to be smooth if the data are smooth. Indeed, very recently, Gutierrez Lanconelli and Montanari [62] proved the following negative regularity result. To state the theorem, we need some more notation. With $B_{r}$ we denote the Euclidean ball in $\mathbb{R}^{2 n+1}$ centered at the origin and with radius $r, K$ denote a function of class $C^{\infty}$ defined on the ball $\left(B_{1} \times \mathbb{R}\right)$, strictly positive and such that $s \mapsto K(\cdot, s)$ is increasing. Then, we have the following result.

Theorem 5.15 (Gutierrez-Lanconelli-Montanari [62]). There exist $r \in(0,1)$ and a pseudoconvex function $u \in \operatorname{Lip}\left(\bar{B}_{r}\right)$ solving

$$
\mathscr{L}^{(n)}(u)=K(x, u) \quad \text { in } \quad B_{r},
$$

in the weak viscosity sense and such that

- $u \notin C^{1}\left(B_{r}\right)$ if $n=2$

- $u \notin C^{1, \beta}$ for any $\beta>1-\frac{2}{n}$ when $n>2$.

This equation is the motivation for a number of interesting problems: symmetry problems and isoperimetric integral inequalities [80] of surfaces with prescribed Levi curvature, and regularity results of radially symmetric solutions.

\section{References}

1. B. Abbondanza, A. Bonfiglioli, On the Dirichlet problem and the inverse mean value theorem for a class of divergence form operators, J. London Math. Soc., 87, 321-346 (2013).

2. D.H. Armitage, S.J. Gardiner, Classical Potential Theory, Springer Monographs in Mathematics, Springer-Verlag, London (2001).

3. J. August, S. Zucker, Sketches with curvature: The curve indicator random field and markov processes, IEEE Trans. Pattern Anal. Mach. Intell, 25(4) (2003), 387-400. 
4. E. Barucci, S. Polidoro, V. Vespri, Some results on partial differential equations and Asian options, Math. Models Methods Appl. Sci., 11 (2001),475-497.

5. E. Battaglia, A. Bonfiglioli, Normal families of functions for subelliptic operators and the theorems of Montel and Koebe, J. Math. Anal. Appl., 409, 1-12 (2014).

6. E.F. Beckenbach, T. Radó: Subharmonic functions and minimal surfaces, Trans, Amer. Math. Soc. 35, (1933), 648661

7. E. Bedford, B. Gaveau, Hypersurfaces with Bounded Levi Form, Indiana University Journal 27 No. 5 (1978), 867-873.

8. E. Bedford, W. Klingenberg, On the envelope of holomorphy of a 2-sphere in $\mathbb{C}^{2}$, J. Amer. Math. Soc. 4 (1991), no. 3, 623-646.

9. W. Blaschke, Ein Mittelwertsatz und eine kennzeichnende Eigenschaft des logarithmischen Potentials, Leipz. Ber., (1916), 68, 37.

10. F. Black and M. Scholes, The pricing of options and corporate liabilities, J. Political Economy, 81 (1973), 637-654.

11. A. Bonfiglioli, E. Lanconelli: Liouville-type theorems for real sub-Laplacians, Manuscripta Math. 105, (2001), 111-124.

12. A. Bonfiglioli, E. Lanconelli, Maximum Principle on unbounded domains for subLaplacians: a Potential Theory approach, Proc. Amer. Math. Soc. 130, (2002), 2295-2304.

13. A. Bonfiglioli, E. Lanconelli, Subharmonic functions on Carnot groups, Math. Ann. 325, (2003), 97-122.

14. A. Bonfiglioli, E. Lanconelli, Dirichlet problem with $L^{p}$-boundary data in contractible domains of Carnot groups, Ann. Sc. Norm. Super. Pisa, Cl. Sci. 5, (2006), 579-610.

15. A. Bonfiglioli, E. Lanconelli: Gauge functions, Eikonal equations and Bôcher's theorem on stratified Lie groups, Calc. Var. Partial Diff. Equations 30, (2007), 277-291.

16. A. Bonfiglioli, E. Lanconelli: A new characterization of convexity in free Carnot groups, Proc. Amer. Math. Soc., 140, (2012), 3263-3273.

17. A. Bonfiglioli, E. Lanconelli, Subharmonic functions in sub-Riemannian settings, J. Eur. Math. Soc., 15, 387-441 (2013).

18. A. Bonfiglioli, E. Lanconelli, A. Tommasoli: Convexity of average operators for subsolutions to subelliptic equations Analysis \& PDE, 7-2, 345-373 (2014). Doi: 10.2140/apde.2014.7.345

19. A. Bonfiglioli, E. Lanconelli, F. Uguzzoni, Uniform Gaussian estimates of the fundamental solutions for heat operators on Carnot groups, Adv. Differential Equations, 7 (2002), 11531192.

20. A. Bonfiglioli, E. Lanconelli, F. Uguzzoni, Fundamental solutions for non-divergence form operators on stratified groups, Trans. Amer. Math. Soc. 356 (2004), no. 7, 2709-2737.

21. A. Bonfiglioli, E. Lanconelli, F. Uguzzoni: Stratified Lie Groups and Potential Theory for their sub-Laplacians, Springer Monographs in Mathematics, 26, New York, NY: SpringerVerlag, 2007.

22. A. Bonfiglioli, F. Uguzzoni, Harnack inequality for non-divergence form operators on stratified groups, Trans. Amer. Math. Soc. 359 (2007), no. 6, 2463-2481.

23. A. Bonfiglioli, F. Uguzzoni, Families of diffeomorphic sub-Laplacians and free Carnot groups, Forum Math. 16, (2004), 403-415.

24. A. Bonfiglioli, F. Uguzzoni, A note on lifting of Carnot groups, Rev. Mat. Iberoamericana 21 (2005), no. 3, 1013-1035.

25. J. M. Bony, Principe du maximum, inégalité de Harnack et unicité du problème de Cauchy pour les opérateurs elliptiques dégénérés, Ann. Inst. Fourier, 19 (1969), 277-304.

26. U. Boscain, S. Polidoro, Gaussian estimates for hypoelliptic operators via optimal control, Rend. Lincei Mat. Appl. Vol. 18, (2007),343-349.

27. M. Bramanti, Singular integrals in nonhomogeneous spaces: $L^{2}$ and $L^{p}$ continuity from Hölder estimates, Revista Matematica Iberoamericana, 26 (2010), no. 1, 347-366.

28. M. Bramanti, L. Brandolini, E. Lanconelli, F. Uguzzoni, Non-divergence equations structured on Hörmander vector fields: heat kernels and Harnack inequalities, Mem. Amer. Math. Soc. 204, no. 961, vi+123 pp. (2010) 
29. M. Bramanti, L. Brandolini, M. Manfredini, M. Pedroni, Fundamental solutions and local solvability for nonsmooth Hrmander's operators, preprint.

30. M. Bramanti, L. Brandolini, M. Pedroni, Basic properties of nonsmooth Hörmander's vector fields and Poincaré's inequality, Forum Math. 25 (2013), no. 4, 703-769.

31. M. Bramanti, G. Cupini, E. Lanconelli, E. Priola, Global $L^{p}$ estimates for degenerate Ornstein-Uhlenbeck operators, Mathematische Zeitschrift, 266 (2010), n. 4, 789-816.

32. M. Bramanti, G. Cupini, E. Lanconelli,, : Global L ${ }^{p}$ estimates for degenerate OrnsteinUhlenbeck operators with variable coefficients Math. Nach., 286 (2013), 10871101.

33. L. Capogna, D. Danielli, N. Garofalo, Subelliptic mollifiers and a basic pointwise estimate of Poincaré type, 226 (1997), no. 1, 147154.

34. L. Capogna, G. Citti, M. Manfredini, Regularity of non-characteristic minimal graphs in the Heisenberg group H1, Indiana Univ. Math. J. 58 (2009), no. 5, 21152160.

35. L. Capogna, G. Citti, M. Manfredini, Uniform Gaussian bounds for sub elliptic heat kernels and an application to the total variation flow of graphs over Carnot groups, Analysis and Geometry in Metric Spaces, 1, (2013), 255275.

36. S. Chapman, T. G. Cowling, The mathematical theory of nonuniform gases, Cambridge University Press, Cambridge, third ed., 1990.

37. C. Cinti, S. Polidoro, Pointwise local estimates and Gaussian upper bounds for a class of uniformly subelliptic ultraparabolic operators, J. Math. Anal. Appl. Vol. 338, (2008), 946969.

38. Cinti C., Menozzi S., Polidoro S., Two-sided bounds for degenerate processes with densities supported in subsets of $R^{N}$ to appear on Potential Anal. (2014).

39. G. Citti, A comparison theorem for the Levi equation, Rend. Mat. Accad. Lincei 4 (1993) 207-212.

40. G. Citti, $C^{\infty}$ regularity of solutions of the Levi equation, Ann. Inst. H. Poincaré Anal. Non Linéaire, 15 (1998), 517-534.

41. G. Citti, N.Garofalo, E. Lanconelli, Harnack's inequality for sum of squares of vector fields plus a potential Amer. J. Math. 115, (1993), 699-734

42. G. Citti, E. Lanconelli, A. Montanari, Smoothness of Lipschitz continuous graphs with non vanishing Levi curvature, Acta Math. 188 (2002), no. 1, 87-128

43. G.Citti, M. Manfredini, F. Serra Cassano, A. Pinamonti, Poincaré-type inequality for intrinsic Lipschitz continuous vector fields in the Heisenberg group, preprint.

44. G.Citti, A.Pascucci, S.Polidoro, On the regularity of solutions to a nonlinear ultraparabolic equation arising in mathematical finance, Differential Integral Equations, 14, (2001), no.6, 701738 .

45. G. Citti, A. Sarti, A cortical based model of perceptual completion in the roto-translation space ournal of Mathematical Imaging and Vision, 24(3), (2006), 307-326.

46. R. Coifman, G. Weiss, Analyse Harmonique Non-Commutative sur Certains Espaces Homogenes. Lecture Notes in Mathematics, n. 242. Springer-Verlag, Berlin-Heidelberg-New York, 1971.

47. C. Constantinescu, A. Cornea, Potential Theory on Harmonic Spaces, Springer-Verlag, (1972)

48. F. Corielli, P. Foschi, and A. Pascucci, Parametrix approximation of diffusion transition densities, SIAM J. Financial Math., 1 (2010), 833-867.

49. F. Da Lio, A. Montanari, Existence and Uniqueness of Lipschitz Continuous Graphs with Prescribed Levi Curvature, Ann. Inst. H. Poincaré Anal. Non Linéaire, 23 (2006), no. 1, $1-28$.

50. D.Danielli, N.Garofalo, D.M. Nhieu, S.D. Pauls, Instability of graphical strips and a positive answer to the Bernstein problem in the Heisenberg group H1, J.Differential Geom., (2009), no. 81, 251295.

51. M. Di Francesco, A. Pascucci, On a class of degenerate parabolic equations of Kolmogorov type, AMRX Appl. Math. Res. Express, (2005), pp. 77-116.

52. M. Di Francesco, S. Polidoro, Schauder estimates, Harnack inequality and Gaussian lower bound for Kolmogorov type operators in non-divergence form, Adv. Diff. Eq. 11 (2006), no. $11,1261-1320$ 
53. M. Escobedo, J.L. Vazquez, E. Zuazua, Entropy solutions for diusion-convection equations with partial diffusivity, Trans. Am. Math. Soc., 343 (1994), 829-842.

54. E. B. Fabes, N. Garofalo Mean value properties of solutions to parabolic equations with variable coefficients, J. Math. Anal. Appl., 121 (1987), no. 2, 305-316.

55. G. B. Folland, Subelliptic estimates and function spaces on nilpotent Lie groups, Arkiv for Mat. 13, (1975), 161-207.

56. P. Foschi and A. Pascucci, Calibration of a path-dependent volatility model: empirical tests, Comput. Statist. Data Anal., 53 (2009), 2219-2235.

57. B. Franchi, E. Lanconelli, Une métrique associée à une classe d'opérateurs elliptiques dégénérés, Rend. Sem. Mat. Univ. Politec. Torino (1983), no. Special Issue, 105-114 (1984), Conference on linear partial and pseudodifferential operators (Torino, 1982).

58. B. Franchi, E. Lanconelli, Hölder regularity theorem for a class of linear nonuniformly elliptic operators with measurable coefficients, Ann. Scuola Norm. Sup. Pisa Cl. Sci. (4) 10 (1983), no. 4, 523-541.

59. B. Franchi, G. Lu, R.L. Wheeden, A relationship between Poincare type inequalities and representation formulas in spaces of homogeneous type, Internat. Math. Res. Notices, (1996),no.1, 114.

60. N. Garofalo, E. Lanconelli, Wiener's criterion for parabolic equations with variable coeffcients and its consequences, Trans. Amer. Math. Soc., 308 (1988), 811-836.

61. N. Garofalo, E. Lanconelli, Asymptotic behavior of fundamental solutions and potential theory of parabolic operator with variable coefficients, Math. Ann., 283, 211239 (1989)

62. C. Gutierrez, E. Lanconelli, and A. Montanari, Nonsmooth Hypersurfaces with Smooth Levi Curvature, Nonlinear Analysis, TMA, 76 (2013), 115-121.

63. D. G. Hobson and L. C. G. Rogers, Complete models with stochastic volatility, Math. Finance, 8 (1998), pp. 27-48.

64. W. Hoh, N. Jacob, Remarks on mean value properties of solutions of second order differential operators, Expo. Math. 9, 367377 (1991)

65. L. Hörmander, Hypoelliptic second order differential equations, Acta Math., 119 (1967), pp. 147-171.

66. D. S. Jerison, A. Sánchez-Calle, Estimates for the heat kernel for a sum of squares of vector fields, Indiana Univ. Math. J. 35 (1986), no. 4, 835-854.

67. A. Kogoj, E. Lanconelli, An invariant Harnack inequality for a class of hypoelliptic ultraparabolic equations Mediterr. J. Math., Vol. 1, 51-80 (2004).

68. A. Kogoj, E. Lanconelli G. Tralli, An inverse mean value property for evolution equations Adv. Differential Equations, Vol. 19, Numbers 7-8 (2014), 783-804.

69. A. Kogoj, G. Tralli Blaschke, Privaloff, Reade and Saks Theorems for diffusion equations on Lie groups, Potential Anal. 38 (2013), no. 4, 1103-1122.

70. A. Kolmogorov, Zufllige Bewegungen. (Zur Theorie der Brownschen Bewegung.)., Ann. of Math., II. Ser., 35 (1934), pp. 116-117.

71. S. Kusuoka, D. Stroock, Long time estimates for the heat kernel associated with a uniformly subelliptic symmetric second order operator, Ann. of Math. (2) 127 (1988), no. 1, 165-189.

72. E. Lanconelli, “Potato Kugel” for Sub-Laplacians, Isr. J. Math., 194 (2013), 277283.

73. E. Lanconelli and D. Morbidelli, On the Poincaré inequality for vector fields, Ark. Mat. 38 (2000), no. 2, 327-342.

74. E. Lanconelli, A. Pascucci, On the fundamental solution for hypoelliptic second order partial differential equations with non-negative characteristic form, Ricerche Mat., 48, (1999), 1, 81-106, 0035-5038,

75. E. Lanconelli, A. Pascucci, Superparabolic functions related to second order hypoelliptic operators, Potential Analysis 11 (1999), 303-323.

76. E. Lanconelli, F. Uguzzoni, Potential analysis for a class of diffusion equations: a Gaussian bounds approach. J. Differential Equations 248, (2010), 2329-2367.

77. A. Lunardi, Schauder estimates for a class of parabolic operators with unbounded coefficients in $R^{n}$, Ann. Scuola Norm. Sup. Pisa (4) 24 (1997), no. 1, 133-164.

78. E. Lanconelli and S. Polidoro, On a class of hypoelliptic evolution operators, Rend. Sem. Mat. Univ. Politec. Torino, 52 (1994), pp. 29-63. 
79. M. Manfredini, The Dirichlet problem for a class of ultraparabolic equations, Adv. Differential Equations, 2 (1997), pp. 831-866.

80. V. Martino, A. Montanari, Integral formulas for a class of curvature PDE's and applications to isoperimetric inequalities and to symmetry problems, Forum Mathematicum 22 (2010) 253-265.

81. A. Montanari, On the regularity of solutions of the prescribed Levi curvature equation in several complex variables. Nonlinear elliptic and parabolic equations and systems (Pisa, 2002). Comm. Appl. Nonlinear Anal. 10 (2003), no. 2, 63-71.

82. A. Montanari, Hölder a priori estimates for second order tangential operators on CR manifolds. Ann. Scuola Norm. Sup. Pisa Cl. Sci (5) Vol. II (2003), pp. 345-378.

83. A. Montanari and F. Lascialfari, The Levi Monge-Ampère equation: smooth regularity of strictly Levi convex solutions, J. Geom. Anal. 14 (2004), no. 2, 331-353.

84. A. Montanari, E. Lanconelli: Pseudoconvex fully nonlinear partial differential operators: strong comparison theorems. J. Differential Equations 202 (2004), no. 2, 306-331.

85. A. Montanari, D. Morbidelli, Step-s involutive families of vector fields, their orbits and the Poincaré inequality, J. Math. Pures Appl. (9) 99 (2013), no. 4, 375-394.

86. R.Monti, F.Serra Cassano, D.Vittone, A negative Answer to the Bernstein Problem for Intrinsic Graphs in the Heisenberg Group, Bollettino U.M.I, 9, 2009.

87. D. Mumford, Elastica and computer vision. In Algebraic geometry and its applications (West Lafayette, IN, 1990), pages 491-506. Springer, New York, 1994.

88. A. Nagel-E. M. Stein-S. Wainger, Balls and metrics defined by vector fields I: Basic properties, Acta Mathematica, 155 (1985), 130-147.

89. P. Negrini, V. Scornazzani, Superharmonic functions and regularity of boundary points for a class of ellipticparabolic partial differential operators, Boll. Unione Mat. Ital. (6) 3 (1984) 85-107.

90. B. Oksendal, Stochastic Differential Equations: An Introduction with Applications, , Springer-Verlag Heidelberg New York, 2003.

91. A. Pascucci, PDE and martingale methods in option pricing, vol. 2 of Bocconi Springer Series, Springer, Milan; Bocconi University Press, Milan, 2011.

92. A. Pascucci and S. Polidoro Harnack inequalities and Gaussian estimates for a class of hypoelliptic operators, Trans. Amer. Math. Soc., Vol. 358 n.11, 4873-4893, (2006)

93. B. Pini, Sulle equazioni a derivate parziali lineari del secondo ordine in due variabili di tipo parabolico, Ann. Mat. Pura Appl. 32 (1951), 179204.

94. I. Privaloff,Sur le fonctions harmoniques, Rec. Math. Moscou (Mat. Sbornik), 32, (1925), 464471.

95. S. Polidoro, A global lower bound for the fundamental solution of Kolmogorov-FokkerPlanck equations, Arch. Rational Mech. Anal., 137 (1997), 321-340.

96. S. Polidoro, On a class of ultraparabolic operators of Kolmogorov-Fokker-Planck type, Matematiche (Catania), 49 (1994), 53-105.

97. C. Rios, E. T.Sawyer, R. L. Wheeden, Regularity of subelliptic Monge-Ampre equations, Adv. Math. 217 (2008), no. 3, 967-1026.

98. L. Rothschild, E.M. Stein, Hypoelliptic differential operators and nilpotent groups, Acta Math., 137 (1976), pp. 247-320.

99. Y.I. Shatyro, Smoothness of solutions of certain singular second order equations, Mat. Zametki 10 (1971), 101-111; English Transl., Math. Notes 10 (1971), 484-489.

100. Z. Slodkowski, G. Tomassini, Weak solutions for the Levi equation and envelope of holomorphy, J. Funct. Anal. 101 (1991), 392-407

101. Z. Slodkowski and G. Tomassini, The Levi equation in higher dimensions and relationships to the envelope of holomorphy, Amer. J. Math. 116 (1994), no. 2, 479-499.

102. E.T. Sawyer, R.L. Wheeden, Regularity of degenerate Monge-Ampere and prescribed Gaussian curvature equations in two dimensions, Potential Analysis, no. 3, 24, (2006), 267301.

103. G. Tomassini, Geometric properties of solutions of the Levi equation, Ann. Mat. Pura Appl.(4) 152 (1988) 331-344

104. G. Tralli, F. Uguzzoni, Wiener criterion for X-elliptic operators, preprint. 
105. F. Uguzzoni, Cone criterion for non-divergence equations modeled on Hrmander vector fields, Subelliptic PDE's and applications to geometry and finance, 227-241, Lect. Notes Semin. Interdiscip. Mat., 6, Semin. Interdiscip. Mat. (S.I.M.), Potenza, 2007.

106. F. Uguzzoni, Estimates of the Green function for X-elliptic operators, Math. Ann. (2014)

107. N. Th.Varopoulos, L. Saloff-Coste, T. Coulhon, Analysis and geometry on groups. Cambridge Tracts in Mathematics, 100. Cambridge University Press, Cambridge, 1992.

108. N.A. Watson, A theory of subtemperatures in several variables, Proc. London Math. Soc.26 (1973), 385417.

109. L. Williams and D. Jacobs, Stochastic completion fields: A neural model of illusory contour shape and salience, In ICCV, 408-415, (1995). 